\title{
Evaluating Lexical Approximation of Program Dependence
}

\author{
Seongmin Lee ${ }^{\mathrm{a}, *}$, David Binkley ${ }^{\mathrm{c}}$, Nicolas Gold ${ }^{\mathrm{b}}$, Syed Islam $^{\mathrm{b}}$, Jens Krinke $^{\mathrm{b}}$, \\ Shin Yoo \\ ${ }^{a}$ KAIST, 291 Daehak-ro, Yuseong-gu, Daejeon 34141, Republic of Korea \\ ${ }^{b}$ University College London, Gower St, London WC1E 6BT, UK \\ ${ }^{c}$ Loyola University Baltimore, 4501 North Charles Street, Baltimore, MD 21210, USA
}

\begin{abstract}
Complex dependence analysis typically provides an underpinning approximation of true program dependence. We investigate the effectiveness of using lexical information to approximate such dependence, introducing two new deletion operators to Observation-Based Slicing (ORBS). ORBS provides direct observation of program dependence, computing a slice using iterative, speculative deletion of program parts. Deletions become permanent if they do not affect the slicing criterion. The original ORBS uses a bounded deletion window operator that attempts to delete consecutive lines together. Our new deletion operators attempt to delete multiple, non-contiguous lines that are lexically similar to each other. We evaluate the lexical dependence approximation by exploring the trade-off between the precision and the speed of dependence analysis performed with new deletion operators. The deletion operators are evaluated independently, as well as collectively via a novel generalization of ORBS that exploits multiple deletion operators: Multi-operator Observation-Based Slicing (MOBS). An empirical evaluation using three Java projects, six C projects, and one multi-lingual project written in Python and $\mathrm{C}$ finds that the lexical information provides a useful approximation to the underlying dependence. On average, MOBS can delete $69 \%$ of lines deleted by the original ORBS, while taking only $36 \%$ of the wall clock time required by ORBS.
\end{abstract}

Keywords: ORBS, Program Slicing, Lexical Analysis

\section{Introduction}

Program slicing often acts as a preprocess for tasks such as testing (Binkley, 1998), debugging (Agrawal et al., 1993), maintenance (Gallagher and Lyle, 1991), and program comprehension (Korel and Rilling, 1998). Traditional program slicing techniques require complex dependence analysis to approximate the underlying dependencies (Binkley et al., 2015). Recently introduced Observation-Based Slicing (ORBS) (Binkley

\footnotetext{
*Corresponding author

Email addresses: bohrok@kaist.ac.kr (Seongmin Lee), binkley@cs . loyola.edu (David Binkley), n.gold@ucl.ac.uk (Nicolas Gold), s.islam@cs.ucl.ac.uk (Syed Islam), j.krinke@ucl.ac.uk (Jens Krinke), shin.yoo@kaist.ac.kr (Shin Yoo)
} 
et al., 2014a; Gold et al., 2017) foregoes this need, discovering the exact dynamic dependencies given a set of inputs. However, this discovery can be expensive since, in the case of statements separated by (large amounts of) other code, many iterations may be required to remove all of the independent code. Given this shortcoming, we conjecture that lexical similarity (e.g., two lines that both include the string tax_rate) might provide a useful approximation to the dependence information. If so, then in the case of ORBS, similar lines can likely be deleted together, irrespective of their relative separation. Thus lexical similarity should bring value in the form of an efficiency gain (assuming that lexical dependence can act as a proxy for the true dependence).

As an example, consider a situation where source lines $l_{1}$ and $l_{2}$ relate to a (single) computation, unrelated to the slicing criterion, but sufficiently far apart from each other that they are never in the same deletion window (the contiguous group of lines selected by ORBS as a deletion candidate). Since they are unrelated to the slicing criterion they can in principle be deleted simultaneously. A lexical approximation of dependence seeks to identify the two as related and therefore potentially deletable in a single step. For example, $l_{1}$ might assign the variable tax_rate, which is subsequently used in $l_{2}$ : tax $=$ tax_rate $*$ sale, while the slicing criterion concerns a variable independent from tax_rate. The lexical connection between $l_{1}$ and $l_{2}$ (i.e., the common occurrence of the words 'tax' and 'rate') might be exploited in an attempt to delete both lines simultaneously.

Exploiting the lexical dependence approximation, techniques that rely on the repeated compilation and execution for their operation should benefit because doing so would permit operations on spatially separated parts of the code. In the case of ORBS, for example, exploiting lexical dependence should enable the deletion of multiple spatially-separated lines in a single compile/execute step. Doing so would permit the full scope of the program to be examined for deletion candidates at any one time rather than just adjacent lines. The net effect would be witnessed as an improvement in slicing performance.

This paper presents and investigates two approaches to the lexical approximation of dependence: one using the Vector Space Model (Salton et al., 1975) and the other using Latent Dirichlet Analysis (LDA) (Blei et al., 2003). To investigate the two, we use them as the basis for two new deletion operators within ORBS. The investigation first considers each new operator in isolation and then in concert with each other and the original ORBS deletion operator.

In the later case, we investigate the possibility of employing multiple deletion operators within ORBS. Ideally, the slicer could select the operator that leads to the largest successful deletion. In the absence of such knowledge, we consider two selection heuristics: Fixed Operator Selection (FOS), which relies on probabilities determined a priori, and Rolling Operator Selection (ROS), which learns the relative applicability of each deletion operator as it computes a slice. The resulting generalization of ORBS uses FOS or ROS to select between multiple deletion operators at each step.

The effectiveness of the lexical dependence approximation is measured using slicing effectiveness (i.e., how small are the resulting slices when compared to the original ORBS slices?) and slicing efficiency (i.e., how fast can the slicing be undertaken in comparison with the original ORBS algorithm?). In other words, what is the trade-off approximating the dependence lexically brings in terms of slice size and slice time. 
We compare the original ORBS implementation (Binkley et al., 2014a) against the new deletion operators in isolation, and their combination with the existing deletion operator, using thirty slicing criteria selected from ten projects written in various programming languages including Java, $\mathrm{C}$, and Python: eighteen slicing criteria from three real world Java projects, six slicing criteria from six C programs taken from the Siemens suite, and six slicing criteria from a multi-lingual open-source project written in Python and C. The results show that slicing using only lexical deletion operators leads to larger slices but take significantly less time. Furthermore, the combined use of the new and existing deletion operators deletes $63 \%$ to $83 \%$ of lines deleted by ORBS in $27 \%$ to $45 \%$ of the time.

This paper makes the following technical contributions:

- We introduce two new lexical dependence approximations.

- To study the two approximations independently we introduce two new variants of observation-based slicing, VSM-ORBS and LDA-ORBS, which each exploit lexical information. Broad stroke, the experiments show that lexical information can be used to complement existing techniques.

- To study the approximations in concert with the original, we introduce MOBS, Multi-operator Observation-Based Slicing, which selects a deletion operator from a set of deletion operators using four different operator selection strategies.

- We empirically evaluate the new lexical dependence approximations using thirty slicing criteria from Java and C projects, and a multi-lingual project written in Python and C. The results show that, on average, MOBS deletes $69 \%$ of the code deleted by ORBS, but requires only $36 \%$ of its execution time.

\section{Observation-Based Slicing}

Based on Weiser's original slicing definition (Weiser, 1979), observation-based slicing (ORBS) dynamically checks whether one or more consecutive lines of program source code can be deleted by observing the impact of their deletion (Binkley et al., 2014a; Gold et al., 2017): if the source code after deletion either fails to compile or does not preserve the value trajectory of the slicing criterion when executed using the given set of inputs, then the deletion is rejected. While ORBS is designed to under-approximate the full semantics of program dependence through test executions, it has surpassed static slicing in some tasks, such as capturing dependencies that static slicers cannot handle (Binkley et al., 2015), and slicing multi-lingual systems (Binkley et al., 2014a), XML-based modelling languages (Gold et al., 2017), as well as Picture Description Languages, which have visual semantics (Yoo et al., 2017).

ORBS takes as input a slicing criteria and a set of inputs. It computes a slice as a series of decisions about whether to accept the deletion of successive source code lines. If, after deleting a line, the program still compiles and preserves the observed behaviour of the slicing criterion for all inputs, then ORBS accepts the deletion. ORBS iterates until no further deletions can be made. ORBS is thus able to uncover the "ground-truth" dependence with respect to the set of inputs. 
One of the drawbacks of finding ground-truth dependence using ORBS is its inefficiency: ORBS requires a large number of compilations and executions. This partly arises because ORBS is relatively naive in its view of a program, working at the level of lines of text, and treating each line as simply deleted or not. Dependence is thus discovered on the basis of the presence or absence of a line, with no regard for its content (thus permitting language independence). Furthermore, considering only single lines limits ORBS ability to delete mutually dependent lines such as the braces around an empty block.

To address the mutually dependent lines problem, the original ORBS implementation (Binkley et al., 2014a), uses a deletion window approach, which enables it to handle sequences of source lines that can only be deleted together (e.g., the pair of brackets that enclose an empty block). Applied to line $l_{i}$, ORBS attempts to delete from one up to $k$ lines (i.e., from $\left\{l_{i}\right\}$ to $\left\{l_{i}, \ldots, l_{i+k-1}\right\}$ ). If it successfully deletes $j$ lines (i.e., $\left\{l_{i}, \ldots, l_{i+j-1}\right\}$ ), the deletion continues with line $l_{i+j}$; if all $k$ attempts fail, the deletion continues with line $l_{i+1}$. Thus after each successful deletion, ORBS moves onto the next target source code line (skipping over the deleted lines), while after each unsuccessful deletion it reverts the deletion before moving on to the next line of the file. ORBS performs multiple passes over the code until it cannot delete anything further. Because in practice it is more efficient, the implementation considers the lines of source code in reverse order, for example, in the hope of deleting all of a variable's uses before attempting the deletion of its declaration.

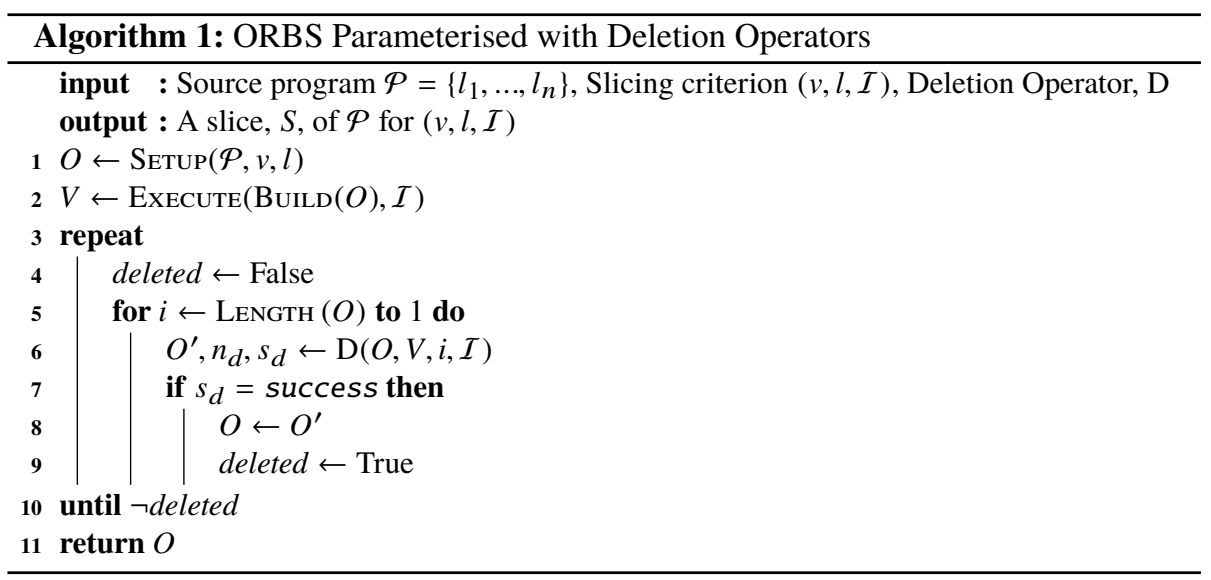

As shown in Algorithm 1, ORBS is parameterised by a deletion operator, D, along with the source program $\mathcal{P}$ and a slicing criterion composed of variable $v$, line $l$, a set of inputs $\mathcal{I}$. In the code the function SETUP inserts probe statements to capture the trajectory of the slicing criterion, while function ExECuTE executes the program with inputs $\mathcal{I}$ and returns the trajectory associated with the slicing criterion.

Deletion operator $\mathrm{D}$ has four parameters: the current observational slice $O$, the original trajectory $V$, the index of the current line $i$, and the inputs $\mathcal{I}$. It first performs a deletion resulting in $O^{\prime}$, and then builds and executes $O^{\prime}$ using $\mathcal{I}$, capturing the trajectory and comparing it with $V$. The operator returns $O^{\prime}$, the number of deleted lines, $n_{d}$, and a 
result of the deletion attempt, $s_{d}$ (either compilation-error, trajectory-change, or success). In the case of a successful deletion, $O^{\prime}$ is made the current observational slice.

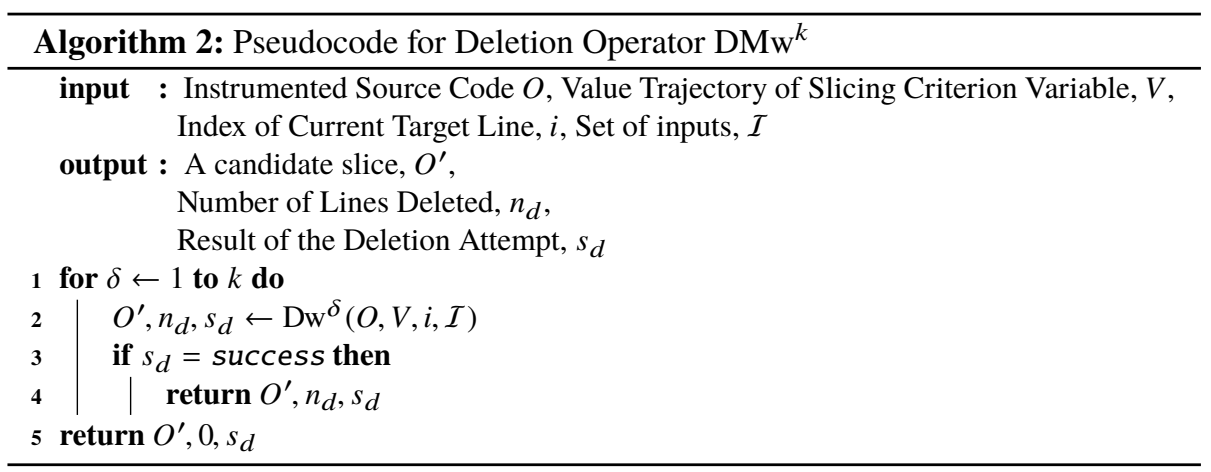

Because it deletes the maximal window up to size $k$, the original ORBS deletion operator, shown as Algorithm 2, is denoted $\mathrm{DMw}^{k}$. This deletion operator is defined in terms of window deletion operator, $\mathrm{Dw}^{\delta}$, which attempts to delete a window of exactly $\delta$ consecutive lines. $\mathrm{DMw}^{k}$ successively applies the $k$ deletion operators, from $\mathrm{Dw}^{1}$ to $\mathrm{Dw}^{k}$, and then returns success if any deletion operator succeeds, immediately, or returns failure if none of $\mathrm{Dw}^{1}$ through $\mathrm{Dw}^{k}$ succeeds. To emphasize its use of a deletion window, we refer to the original ORBS algorithm, which implicitly uses $\mathrm{DMw}^{k}$, as W-ORBS.

\section{Lexical Similarity Deletion Operators}

This section introduces the two new lexical deletion operators that seek to exploit lexical similarity within the text of a program. Lexical similarity can be a good proxy for syntactic or semantic similarity (Ragkhitwetsagul et al., 2018). Our new deletion operators are based on the intuition that if a source line can be safely deleted with respect to a given slicing criterion, then there are likely other lexically similar lines that can also be safely deleted. For example, if the slicing criterion involves a variable that holds an account balance in a banking system, then a line that handles logging will be deleted by ORBS. When deleting this line, it may be beneficial to attempt to delete all other lines that include the lexical token log. In other words, we posit that we can approximate the semantics of program dependence using lexical similarity. To this end we introduce the two lexical deletion operators: Dvsm and DLDA.

The first lexical deletion operator, Dvsm, makes use of the Vector Space Model (VSM), which has been used in Information Retrieval (IR) to calculate the distances between a collection of text documents and a query (Salton et al., 1975). VSM represents each document and the query as a vector of weights, that associate a value with each unique term (word) that occurs in any of the documents. The distance between vectors captures the similarity between the documents and the query.

In our application, $\operatorname{DvsM}^{\gamma}$ is parameterized by a threshold $\gamma$ and treats all lines of text in the source code as individual documents. It identifies two lines as similar 
if their lexical similarity is greater than $\gamma$. In greater detail, let $L$ be the set of all non-comment lexical tokens in the code and let $K$ be a list of stop words typically composed of programming-language reserved keywords. The vocabulary used is the set of terms, $T=L \backslash K$. A range of techniques for assigning term weights and computing distances has been used in VSMs (Mitra and Chaudhuri, 2000). We use tf-idf to determine term weights (Rajaraman and Ullman, 2011) and measure distances using cosine similarity (Singhal, 2001).

The second lexical deletion operator, DLDA, makes use of Latent Dirichlet Allocation (LDA), which models a collection of documents using two probability distributions: each document is represented as a probability distribution of topics where each topic is a probability distribution over the words of the vocabulary (Blei et al., 2003). The similarity between two documents is measured as the distance between their corresponding topic vectors. Similar to $\operatorname{Dvsm}^{\gamma}$, the deletion operator DLDA ${ }^{\gamma}$ treats each source code line as a document, uses the same vocabulary, and also computes distances using cosine similarity.

\section{Variants of ORBS}

Our empirical investigation is designed to investigate the effectiveness of using lexical dependence approximation. To do so, we first introduce two variants of ORBS based on the two lexical deletion operators, Dvsm and DLDa. We then consider two additional variants that, analogous to the use of multiple window sizes by $\mathrm{DMw}^{k}$, each consider a range of parameter (threshold) values. Looking ahead, the empirical analysis considers two final variants (bringing the total to six). These final two follow lexical deletion with the application of W-ORBS. Finally, having a range of deletion operators suggests the potential in trying the leverage the strengths of each. Thus the bulk of this section presents MOBS, Multi-operator Observational Slicing, which aims to selectively apply a range of deletion operators.

\subsection{Lexical ORBS variants}

The first two variants of ORBS are based on Algorithm 1 with $D$ being passed one of the two lexical deletion operators. We refer to ORBS when using Dvsm ${ }^{\gamma}$ as VSM-ORBS. Similarly, we refer to ORBS when using the deletion operator DLDA ${ }^{\gamma}$ as LDA-ORBS. By virtue of using lexical deletion operators, VSM-ORBS and LDAORBS share a few distinguishing features that may yield advantages over W-ORBS.

1. There is no limit to the number of lines that can be deleted in a single deletion.

2. They can delete non-consecutive lines.

3. Because Dvsm and DLDA are lexical, ORBS language independence is preserved.

4. During a single iteration, only one deletion is attempted at each slicing point, unlike W-ORBS, which may attempt multiple deletions at each slicing point depending on the window size.

The other two variants successively apply a deletion operator with a range of parameter values analogous to Dw's successive application of a range of window sizes. Considering first VSM, Algorithm 3 shows the pseudo code for the successive 


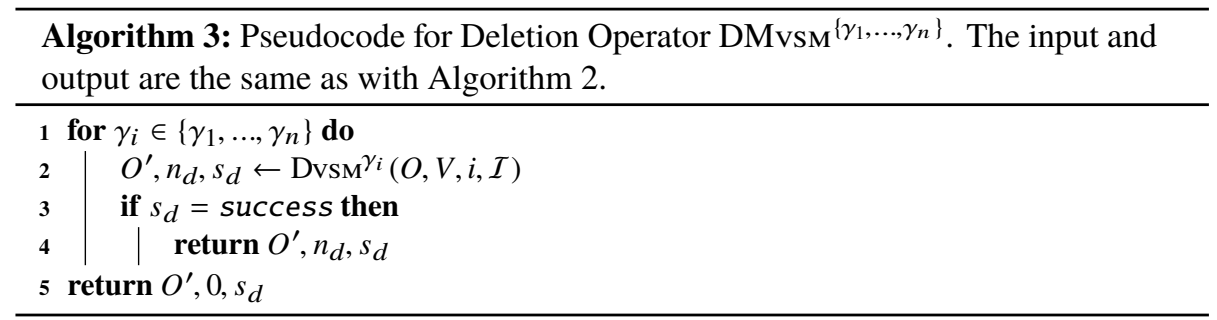

application of Dvsm ${ }^{\gamma}$ for a range of values of $\gamma$ to target source code line, $i$. We denote this deletion operator as $\mathrm{DMvsm}^{\Gamma}$, where $\Gamma$ is a list of thresholds (parameter values). While it may spend more than one deletion attempt on a single line, DMvsм ${ }^{\Gamma}$ thoroughly checks the lexical dependence the line could have with different thresholds. We refer to ORBS when using multiple thresholds with $\operatorname{DMvsm}^{\Gamma}$ as VSM-ORBS-M. Likewise, we refer to ORBS when using DMLDA ${ }^{\Gamma}$ as LDA-ORBS-M.

\subsection{MOBS: Multi-operator Observational Slicing}

With three deletion operators, Dw, DvSM, and DLDA, we can instantiate Algorithm 1 in different ways producing multiple ORBS' variants. However, because each deletion operator attempts to delete different parts of the code, a more synergistic approach might better exploit the strengths of each operator. Here our goal is to improve slicing performance by using the 'right' deletion operator at the 'right' time and in the 'right' place. To study the range of possibilities we introduce MOBS: Multi-operator Observational Slicing, which selectively applies multiple deletion operators while slicing.

\subsubsection{MOBS Algorithm}

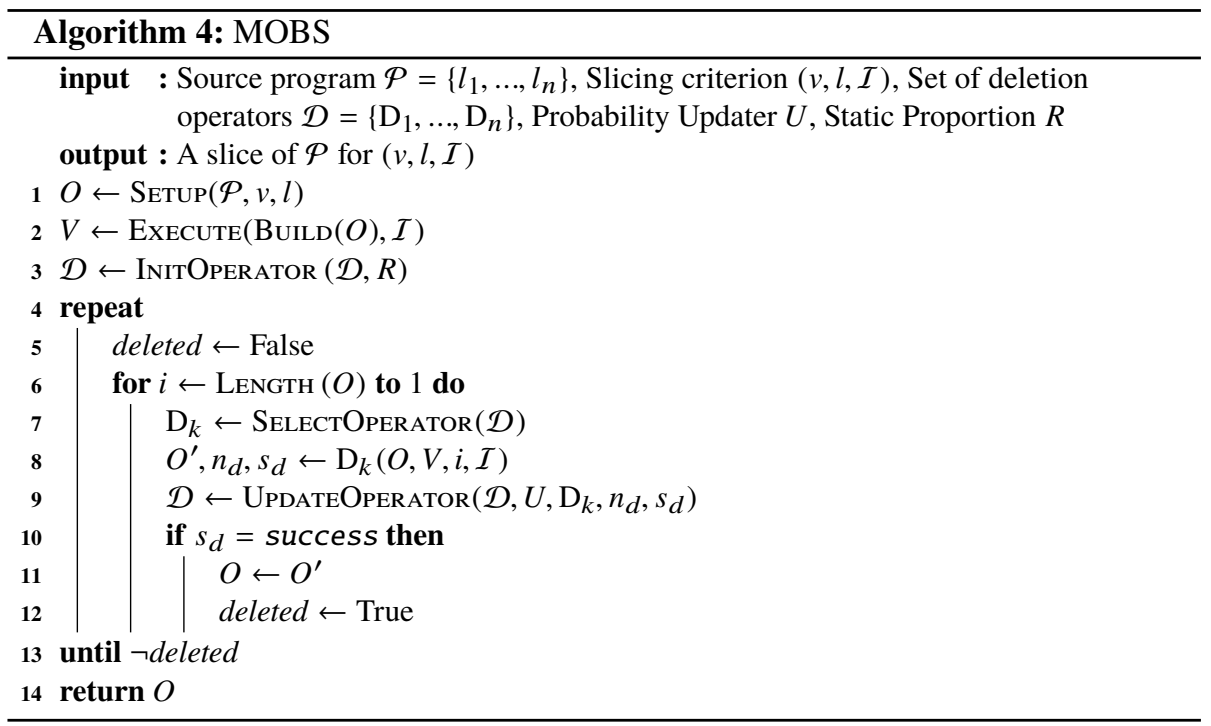


Algorithm 4 presents MOBS, which has the same basic structure as Algorithm 1. MOBS makes use of three helper functions. The first of these, INITOPERATOR, initializes the deletion operator selection probabilities. The second, SelectOperator, chooses a deletion operator to apply at each line using roulette-wheel selection (Goldberg, 1989) based on the probability distribution on operators. Once chosen, the speculative deletion by MOBS is the same as that done by ORBS except that UPDATEOPERATOR updates the probability distribution according to the updater function $U$.

\subsubsection{Fixed Operator Selection (FOS)}

The remainder of this section considers two operator selection strategies: Fixed Operator Selection (FOS) and Rolling Operator Selection (ROS). For a given slicing criterion FOS computes fixed probabilities based on the success proportion of each deletion operator and stores them in $\mathcal{D}$ in Line 3 of Algorithm 4. Such an exhaustive approach is not viable in production where an approximation over multiple criteria would be required, but for the experimental evaluation it serves to establish an upper bound on FOS performance.

We use two methods to compute the success proportion of an operator: the number of successful deletions and the number of lines deleted. We call the proportions calculated by each method its 'applicability' and 'effect', respectively. In addition, we study the use of a uniform proportion as a baseline. Note that for FOS the probabilities remain constant throughout slicing. In other words, the probability updater $U$ is the identity function.

Algorithm 5 details the calculation of the initial probabilities. It can compute applicability (if Line 8 is used) or effect (if Line 9 is used). Given a source program, a slicing criterion, and a set of deletion operators, $\mathcal{D}$, this algorithm returns a proportion array, $R$, for each deletion operator $\mathrm{D}_{k} \in \mathcal{D}$. The function Initialize first assigns each entry the value 0 . The algorithm then iteratively applies each operator $\mathrm{D}_{k}$ to each source line and records in the proportion array $R_{\mathrm{D}_{k}}$, either the deletion's successful application (when using Line 8) or the number of lines deleted (when using Line 9). INITOPERATOR, used in Line 3 of Algorithm 4, takes the proportion array and initializes the selection probability of each deletion operator $\mathrm{D}_{k}$ as follows:

$$
P\left(\mathrm{D}_{k}\right)=\frac{\sum_{1 \leq i \leq n} R_{\mathrm{D}_{k}}[i]}{\sum_{\mathrm{D}_{j} \in \mathcal{D}} \sum_{1 \leq i \leq n} R_{\mathrm{D}_{j}}[i]}
$$

\subsubsection{Rolling Operator Selection (ROS)}

In contrast to the FOS strategy, the Rolling Operator Selection, ROS, updates the probability after each deletion attempt. The intuition here is that early on different operators will be effective than when the slice approaches its final state. The probability distribution over the operators $P\left(\mathrm{D}_{k}\right)$ is initialized with a uniform distribution. UPDATEOPERATOR, first, changes the probability of the current deletion operator using the probability updater $U$ with respect to the result of the deletion attempt. Then, it normalizes the sum of the probability distribution to be 1 .

In our study, we used Equation 2 as the probability updater $U$. The penalty factor for a compilation failure, $\omega_{\text {comp }}$, and an execution failure $\omega_{\text {exec }}$, both range from zero 


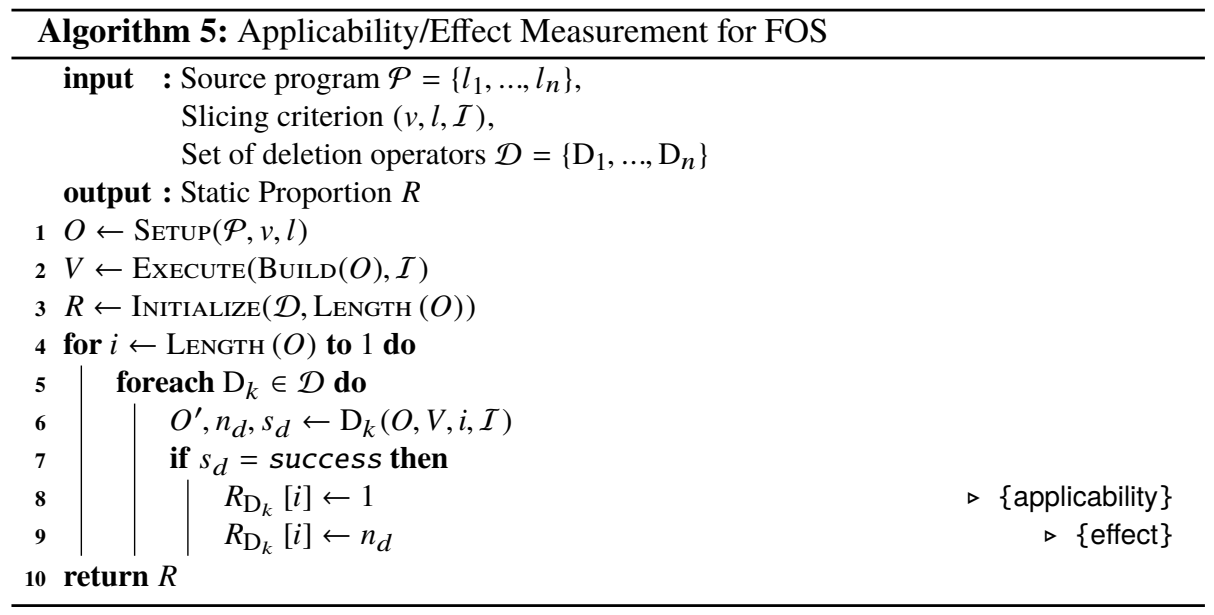

to one. We penalise compilation failure more severely (i.e., $\omega_{\text {comp }} \leq \omega_{\text {exec }}$ ) because successful compilation is necessary for a successful deletion. On the other hand, a successful deletion always increases the probability as $\log _{10}\left(n_{d}+1\right)>0$. Based on our empirical investigation, we set $\omega_{\text {comp }}$ as 0.98 and $\omega_{\text {exec }}$ as 0.99 . The selection of the next deletion operator makes use of the updated distribution.

$$
P_{\text {new }}\left(\mathrm{D}_{k}\right)= \begin{cases}\omega_{\text {comp }} \cdot P\left(\mathrm{D}_{k}\right) & \text { compilation-error } \\ \omega_{\text {exec }} \cdot P\left(\mathrm{D}_{k}\right) & \text { trajectory-change } \\ \left(1+\log _{10}\left(n_{d}+1\right)\right) \cdot P\left(\mathrm{D}_{k}\right) & \text { success }\end{cases}
$$

\section{Research Questions}

We investigate the following six research questions:

RQ1. Lexical Deletion Operators: How efficient and how effective are the lexical deletion operators?

We compare the results of VSM-ORBS, LDA-ORBS, and W-ORBS with respect to the number of lines deleted (effectiveness) and the time taken to compute a slice (efficiency). We also investigate the impact of the similarity threshold parameter $\gamma$ of the deletion operators DvSM${ }^{\gamma}$ and DLDA ${ }^{\gamma}$ used by VSM-ORBS and LDA-ORBS, respectively. Finally, we consider the impact of successive application of a range of different thresholds using VSM-ORBS-M and LDA-ORBS-M.

RQ2. Operator Comparison: How different are the deletion operators from each other, both quantitatively and qualitatively?

We compare the lexical dependence approximation provided by the two lexical deletion operators by comparing various statistics gathered when applying each operator to each line of the source code.

RQ3. Operator Selection Strategy: What impact does the operator selection strategy have on MOBS's ability to exploit lexical dependence? 
In contrast to RQ2's head-to-head comparison, RQ3 begins the investigation into how the deletion operators complement each other. Our goal here is to determine which selection strategy to use in the subsequent experiments.

RQ4. Strategy Impact: How does MOBS using the chosen selection strategy compare with W-ORBS?

RQ4 compares MOBS with its best strategy (as determined when considering RQ3) against the original ORBS algorithm, W-ORBS. We again compare the results in terms of effectiveness and efficiency.

RQ5. Qualitative Analysis: What impact do differences in the lexical dependencies considered have on the resulting slices?

To provide a more intuitive feel for the impact of lexical dependence, we investigate characteristics of the slices produced by variants of ORBS and MOBS. The comparison considers several qualitative properties of the resulting slices.

RQ6. Scalability: How well does the lexical dependency approximation scale?

The lexical deletion operators preserve the language agnostic nature of ORBS. With this research question, we investigate the scalability of lexical deletion operators using a larger, multi-lingual program.

\section{Experimental Setup}

\subsection{Metrics}

We define several performance metrics for use in the quantitative analysis. The first three, CPD (Compilations Per Deletion), EPD (Executions Per Deletion), and TPD (Time Per Deletion) capture the efficiency of a slicing method. For these metrics, the smaller the value, the better. On the other hand, DPS (Deletions Per Success) is the number of deleted lines per one successful application of the deletion operator. DPS evaluates the efficiency of a deletion operator: the larger the value, the better (the more efficient the operator).

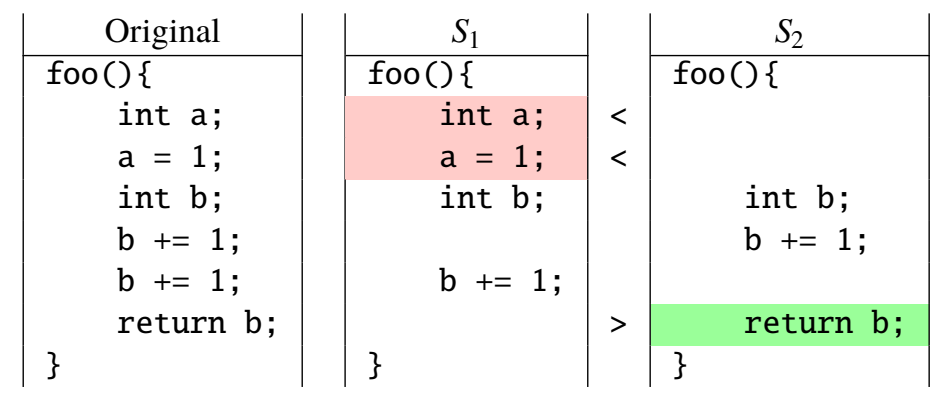

Figure 1: Diff result of two slices of the original code. Our modified Jaccard similarity between $S_{1}$ and $S_{2}$ is: $\operatorname{Jaccard}\left(S_{1}, S_{2}\right)=4 /(4+2+1) \approx 0.57$.

Finally, we use a variant of Jaccard similarity to calculate the similarity between two slices. The traditional definition of Jaccard similarity, which is a measure of 
similarity between two sets, is not appropriate for our purpose because, from the lexical viewpoint, a slice is an ordered multiset, i.e., the order of the lines matter and it may contain multiple instances of the same line. Furthermore, it is not viable to calculate the Jaccard similarity by making each line uniquely identifiable (e.g., by adding the tuple (file name, line number)). Figure 1 shows an example of two slices, $S_{1}$ and $S_{2}$ where $S_{1}$ and $S_{2}$ delete 'b $+=1$;' from different locations, but deletions result in the same common subsequence ' $f \circ o()\{$, int $b ;, b+=1 ;$,$\} '. Adding unique identifiers to$ lines would have unwontedly not produced the same result.

Given two sequences, $S_{1}$ and $S_{2}$, our variant of Jaccard similarity, modified for ordered multisets, is defined as follows:

$$
\operatorname{Jaccard}\left(S_{1}, S_{2}\right)=\frac{|C|}{|C|+\left|O_{1}\right|+\left|O_{2}\right|}
$$

where $C$ as the longest common subsequence ${ }^{1}$ of $S_{1}$ and $S_{2}, O_{1}=S_{1}-C$, and $O_{2}=S_{2}-C$.

\subsection{Subjects and Environment}

Table 1 shows the programs chosen for our empirical evaluation. For Java, we choose three open-source projects: commons-cli ${ }^{2}$ and commons-csv ${ }^{3}$ from Apache Commons Project, and guava ${ }^{4}$, which is a core Java library developed by Google. We choose five slicing criteria from commons-cli, three from commons-csv, and ten from guava (five each from com.google.common.escape and com.google.common.net). For the $\mathrm{C}$ code, we choose the Siemens suite (Do et al., 2005). The program tcas has been excluded from the experiment, as it was too small for the lexical similarity models such as LDA to be applicable (tcas has only 120 Non-Comment Lines of Code (NCLOC). We choose one slicing criterion for each $\mathrm{C}$ program. Finally, the table provides the size of each subject program in NCLOC, as well as the number of test cases provided by the developers. The provided test cases will be used as inputs as part of the slicing criteria. The second from the last row shows the statistics of misaka, an open-source multi-lingual benchmark used to study RQ6. misaka includes both C and Python source code and has a total of over 5,000 NCLOC.

To avoid the task of generating an obvious slice, we choose all slicing criteria thoroughly, making the dependency analysis challenging enough. Each slicing criterion consists of a variable located at a call depth of at least three. For Java, the slicing criteria are chosen from the class which has a dependency with at least three (seven on average) other classes.

For the purposes of this investigation, we filter out comments and reserved words prior to analysis. Although this violates language independence to some degree, it does not demand even the construction of a program's parse trees but is restricted

\footnotetext{
${ }^{1}$ Note that a longest common subsequence refers to a non-consecutive subsequence, such as ' $f \circ o()\{$, int $\mathrm{b} ;, \mathrm{b}+=1 ;$,$\} ' in S_{2}$ of Figure 1 , whereas a longest common substring is a consecutive substring that is common to two strings.

${ }^{2}$ https://commons. apache.org/proper/commons-cli/

${ }^{3}$ https://commons . apache.org/proper/commons-csv/

${ }^{4}$ https://github.com/google/guava
} 


\begin{tabular}{llrrrr}
\hline Lang. & Proj. & \# of Files & NCLOC & \# of Test cases & \# of Slicing Criteria \\
\hline \multirow{4}{*}{ Java } & commons-cli & 23 & 2,081 & 26 & 5 \\
& commons-csv & 11 & 1,504 & 13 & 3 \\
& guava-escape & 10 & 590 & 6 & 5 \\
& guava-net & 9 & 1,569 & 8 & 5 \\
\hline \multirow{4}{*}{$\mathrm{C}$} & prttok & 1 & 410 & 11 & 1 \\
& prttok2 & 1 & 387 & 10 & 1 \\
& replace & 1 & 508 & 15 & 1 \\
& sched & 1 & 208 & 6 & 1 \\
& sched2 & 1 & 276 & 6 & 1 \\
\hline \multirow{2}{*}{ C + Python } & totinfo & 1 & 261 & 6 & 6 \\
& misaka & $(15$ & 5,125 & 92 & 30 \\
\hline \multirow{2}{*}{} & Total & $5)$ & $(4742+473)$ & & \\
\hline
\end{tabular}

Table 1: Subject programs and slicing criteria. The notation $(A+B)$ for misaka denotes the statistics for its C and Python source code, respectively.

to the lexical analysis in the matching of (regular expression) tokens to the stoplist. Consideration of all program elements forms part of our future work.

Experiments were performed on machines with Intel Core i7-6700K and 32GB RAM, running Ubuntu 14.04.5 LTS. Operator specific variants of ORBS (W-, VSM-, and LDA-) as well as MOBS have been implemented and executed in Python version 3.6.5. Java subjects have been built and executed using Java version 1.8.0_141 and JUnit version 4.12. C subjects have been built using GCC version 4.8.4.

\subsection{Configuration}

W-ORBS has a single parameter, $\delta$, the maximum size of the deletion window. Our prior empirical study with W-ORBS has found that using four as the maximum window size provides the best performance. Thus, we use W-ORBS with maximum window size $\delta=4$ as the baseline. While the studied subject programs and their test suites are deterministic, during the slicing process ORBS can produce nondeterministic candidate slices due to changes in control flow that arise from the deletions. Looking ahead, the impact of this non-determinism can be seen in Table 7 where the standard deviation of the number of deleted lines, $\sigma_{\text {del }}$ by W-ORBS is non-zero in some cases. To account for this, as well as the randomness from the use of wall clock execution time, each W-ORBS slice was computed ten times.

Both VSM-ORBS and LDA-ORBS are parameterised by similarity threshold $\gamma$. Since cosine similarity is used, the similarity is in the range of $[0,1]$. We report results using thresholds of 0.6, 0.7, 0.8, and 0.9. LDA-ORBS also requires selecting a topic count, which determines how many topics exist in the model. The best topic count depends heavily on properties such as the size and vocabulary of the documents. Tuning it typically requires manual inspection. We evaluated the values $25,50,100$, $300,500,700$, and 900 during the experiment for RQ1 and choose 500 as the best performer for Java projects; we also evaluated the values $25,50,75,100,200$, and 300 
for $\mathrm{C}$ projects, and chose 200 as the best performer (see Section 7.1 for more details). We set the LDA hyperparameters, $\alpha$ and $\beta$, which affect the sparsity of the documenttopic and topic-word distributions of the LDA model, respectively, to the inverse of the topic count. Finally, for VSM-ORBS-M and LDA-ORBS-M we use two sequences for $\Gamma:\{0.6,0.7,0.8,0.9\}$ and $\{0.9,0.8,0.7,0.6\}$, which we refer to as increasing order and decreasing order. For LDA-ORBS-M we use the same topic count as used with LDAORBS. Note that there is no need to repeat the VSM-ORBS(-M) and LDA-ORBS(-M) runs because VSM-ORBS is deterministic and LDA-ORBS is deterministic apart from the generation of the topic model. Our experience is that the variance from other parameters, such as the similarity threshold and topic count, is much more significant than the variation from rerunning the topic modelling.

The library of deletion operators used by MOBS includes the following twelve operators, which are different paramaterisations of the operators Dw, DvSM, and DLDA:

- $\mathrm{Dw}^{\delta}$ for deletion window size $\delta=1,2,3$, and 4

- $\operatorname{Dvsm}^{\gamma}$ for threshold $\gamma=0.6,0.7,0.8$, and 0.9

- DLDA $^{\gamma}$ for threshold $\gamma=0.6,0.7,0.8$, and 0.9

Due to the stochastic nature of the operator selection, and the use of wall clock time, like W-ORBS, we repeat each MOBS slice ten times for each slicing criterion.

\section{Results}

\subsection{Lexical Deletion Operators}

Before comparing the efficiency and effectiveness of VSM-ORBS, LDA-ORBS, and W-ORBS, we investigate how lexical deletion operators delete source code. Table 2 shows several example deletions. The first criterion of commons-cli, cli-1, involves the program's option-setting function. Dvsm simultaneously deletes lines that are related to the option printing, but are irrelevant to the criterion. In the second example, Dvsm deletes all function calls that handle a deficient token error from prttok2, since the criterion checks whether the input token is an identifier. Similar to cli-1, for the criterion csv-1, DLDA deletes all string building functions from commons-csv, because they are unrelated to the line-break checking function for a csv file.

To answer RQ1, we report results from comparisons between VSM-ORBS, LDAORBS and W-ORBS. As an example, Figure 2 shows the comparison between the three slicers for guava-escape ${ }^{5}$ : the $x$-axis indicates the variant of ORBS ( $\gamma$ denotes the threshold; $n$ the topic count of LDA). On the left is a bar chart showing the number of deleted lines (blue), the number of compilations (light grey), and the number of executions (dark grey), required by each slicer. On the right is a bar chart showing the wall-clock time (red), and the number of lines deleted (blue) for each slicer. We also report the performance metrics CPD (denoted by $\bigcirc$ ), EPD $(\times)$, and TPD $(\diamond)$ with

\footnotetext{
${ }^{5}$ Plots for other slicing criteria as well as other RQs are available at https://coinse.github.io/ MOBS_data_webpage/.
} 


\begin{tabular}{|c|c|c|}
\hline & File-name:Line-num & Code line \\
\hline 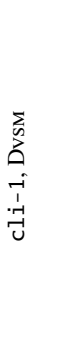 & $\begin{array}{l}\text { HelpFormatter.java:166 } \\
\text { HelpFormatter.java: } 173 \\
\text { HelpFormatter.java:182 } \\
\text { HelpFormatter.java:186 } \\
\text { HelpFormatter.java:191 } \\
\text { HelpFormatter.java:203 } \\
\text { OptionGroup.java:46 } \\
\text { OptionGroup.java:50 } \\
\text { OptionGroup.java:53 } \\
\text { OptionGroup.java:57 } \\
\text { OptionGroup.java:61 } \\
\text { OptionGroup.java:64 }\end{array}$ & $\begin{array}{l}\text { buff.append(" "); } \\
\text { buff.append("["); } \\
\text { buff.append(" I "); } \\
\text { buff.append("]"); } \\
\text { buff.append("["); } \\
\text { buff.append("]"); } \\
\text { buff.append("["); } \\
\text { buff.append("-"); } \\
\text { buff.append("-"); } \\
\text { buff.append(" "); } \\
\text { buff.append(", "); } \\
\text { buff.append("]"); }\end{array}$ \\
\hline 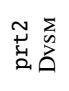 & $\begin{array}{l}\text { print_tokens } 2 . c: 123 \\
\text { print_tokens } 2 . c: 130 \\
\text { print_tokens2.c: } 142\end{array}$ & $\begin{array}{l}\text { unget_error }(t p) \text {; } \\
\text { unget_error }(t p) ; \\
\text { unget_error }(t p) ;\end{array}$ \\
\hline 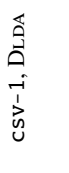 & $\begin{array}{l}\text { CSVFormat.java: } 410 \\
\text { CSVFormat.java: } 416 \\
\text { CSVFormat.java: } 428 \\
\text { CSVFormat.java: } 443 \\
\text { CSVFormat.java: } 447\end{array}$ & $\begin{array}{l}\text { sb. append("Delimiter=<") . append(delimiter) . append('>'); } \\
\text { sb. append (','); } \\
\text { sb. append(' '); } \\
\text { sb. append ("HeaderComments:") } \\
\quad \text {. append(Arrays.toString(headerComments)); } \\
\text { sb. append("Header:"). append(Arrays.toString(header)); }\end{array}$ \\
\hline
\end{tabular}

Table 2: Lines that have been deleted by lexical deletion operators. cli-1 and csv-1 represent the first criterion of commons-cli and commons-csv. prt2 represents the criterion of prttok2.

connected lines. Both VSM-ORBS and LDA-ORBS delete many fewer lines, but with significantly higher efficiency, as can be seen in their lower CPD, EPD, and TPD values: a similar trend is observed across all subjects.

The data in Table 3 compares the efficiency of W-ORBS, VSM-ORBS, and LDAORBS. For these results, the similarity threshold of VSM-ORBS and LDA-ORBS is set to 0.9 ; the topic count for LDA-ORBS is set to 500 for Java projects and 200 for $\mathrm{C}$ projects (the remainder of this Section explains the rationale behind these choices). On average, VSM-ORBS and LDA-ORBS delete $42.2 \%$ and $33.2 \%$ of the number of lines deleted by W-ORBS, respectively. However, VSM-ORBS uses only $19.4 \%$ of compilations and $29.2 \%$ of executions used by W-ORBS, requiring only $21.0 \%$ of the wall clock execution time of W-ORBS. Similarly, LDA-ORBS uses $19.4 \%$ of compilations, $22.1 \%$ of executions, and takes only $20.0 \%$ of the wall clock execution time of W-ORBS.

The results in Table 3 can be summarised using the per deleted line efficiency metrics as follows. For VSM-ORBS, its CPD, EPD, and TPD values are, on average across all subjects, $45.9 \%, 63.1 \%$, and $49.5 \%$ of the corresponding W-ORBS's value. For LDA-ORBS they are $59.9 \%, 65.9 \%$, and $62.2 \%$, respectively.

Figure 3 shows the results of VSM-ORBS with various similarity thresholds. As the threshold increases, the number of deleted lines tends to increase: that is, as VSMORBS targets similar lines with higher thresholds, it becomes more likely that it can delete those lines together. On the other hand, the number of compilations is relatively stable because compilation is performed every time ORBS attempts a deletion. Since the number of compilations is much larger than that of executions, the wall clock execution time tends to follow the trends of compilations. Consequently, the CPD and TPD values show similar trends and are dependent on how the number of deleted lines changes. If the number of deleted lines varies significantly (as in commons-cli), CPD 
guava-escape criterion3: W- and VSM-, LDA-ORBS

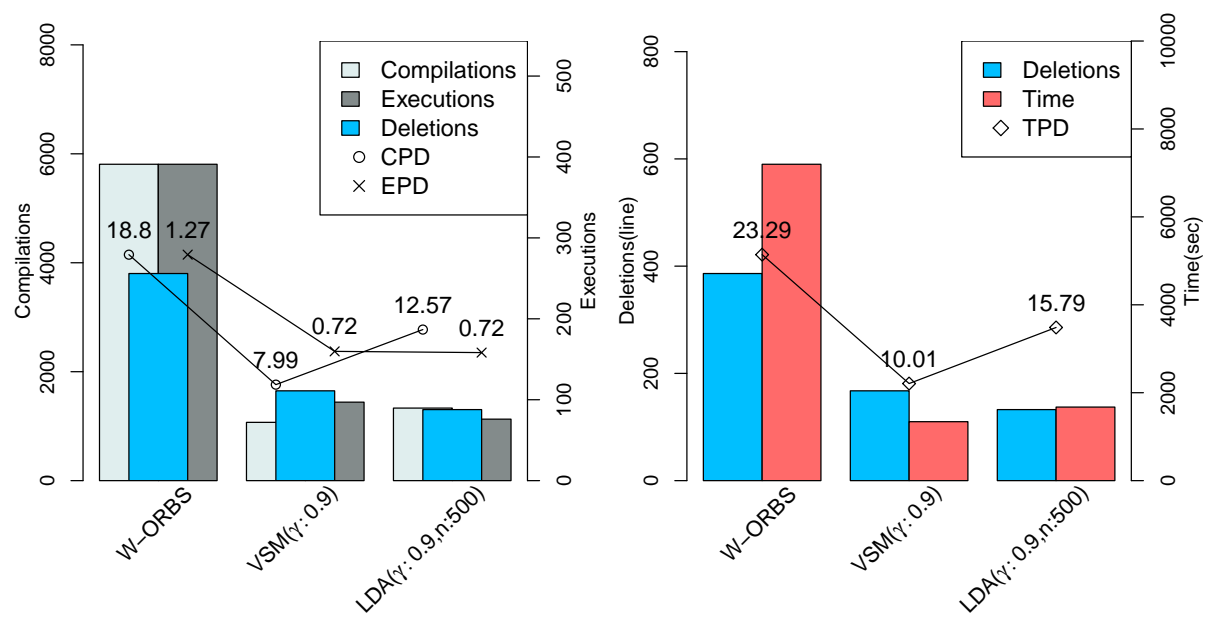

Figure 2: Efficiency of ORBS variants. CPD, EPD, and TPD are the number of compilations, executions, and time taken per deleted line, respectively.

and TPD tend to increase; if the number of deletions varies little (as in commons-csv), CPD and TPD tend to be more stable.

Figure 4 shows the results of LDA-ORBS with various threshold parameters. Overall, the results show similar trends to those of VSM-ORBS: the smaller the threshold value, the worse the efficiency. However, for $\mathrm{C}$ projects, the difference between the number of the compilations and executions is less than that of the Java projects; their compilation time is also smaller. Consequently, the trend of TPD is more likely to follow the EPD.

Let us briefly discuss the impact of topic count on LDA-ORBS. Figure 5 shows the results of LDA-ORBS with various topic counts for commons-cli. In commons-cli, we observe the number of deleted lines increases until it reaches a maximum at topic count of $n=500$, where it levels off. This is because, when $n$ is too small, the topic model cannot capture sufficient features of the source code lines resulting in insufficient similarity and, consequently, fewer deleted lines. A similar trend is observed in the $\mathrm{C}$ subjects: the number of deleted lines increases as $n$ brows to 200, and then levels off. Based on these observations, we use the topic counts 500 for Java and 200 for $\mathrm{C}$ in the remainder of our experiments, as these are the values around which the number of deletions improves and remains stable afterwards.

Finally, we consider the performance of VSM-ORBS-M and LDA-ORBS-M. Figure 6 compares W-ORBS, VSM-ORBS with Dvsm ${ }^{0.9}$, and VSM-ORBS-M using the increasing and decreasing values for $\Gamma$. Results suggest that there is almost no difference in the number of lines deleted by VSM-ORBS and VSM-ORBS-M using either order. VSM-ORBS-M deletes only $0.8 \%$ and $0.5 \%$ more lines than VSM-ORBS with $\Gamma$ increasing and decreasing, respectively. Meanwhile, VSM-ORBS-M (with either $\Gamma$ ) employs $49 \%$ more compilations than VSM-ORBS; thus, CPD increases $48 \%$ when compare to VSM-ORBS. LDA-ORBS and LDA-ORBS-M show a similar pattern; LDA- 


\begin{tabular}{|c|c|c|c|c|c|c|c|c|c|c|c|c|}
\hline \multirow{2}{*}{ Criteria } & \multicolumn{4}{|c|}{ W-ORBS } & \multicolumn{4}{|c|}{ VSM-ORBS } & \multicolumn{4}{|c|}{ LDA-ORBS } \\
\hline & $\mathrm{C}$ & E & $\mathrm{T}$ & D & $\mathrm{C}$ & $\mathrm{E}$ & $\mathrm{T}$ & $\mathrm{D}$ & $\mathrm{C}$ & $\mathrm{E}$ & $\mathrm{T}$ & $\mathrm{D}$ \\
\hline $\mathrm{cli}-1$ & 21052 & 2413 & 28975 & 980 & 3680 & 470 & 5510 & 325 & 4330 & 408 & 5596 & 240 \\
\hline cli-2 & 22241 & 2318 & 25168 & 1123 & 3297 & 370 & 3920 & 375 & 4076 & 359 & 4813 & 291 \\
\hline cli-3 & 21498 & 1886 & 26165 & 1160 & 3583 & 391 & 4805 & 393 & 4195 & 359 & 5535 & 290 \\
\hline cli-4 & 23163 & 2760 & 32246 & 818 & 4472 & 579 & 7005 & 285 & 4306 & 478 & 6178 & 217 \\
\hline cli-5 & 24340 & 2463 & 30812 & 1144 & 3571 & 368 & 4594 & 364 & 4197 & 348 & 5119 & 279 \\
\hline $\mathrm{csv}-1$ & 14627 & 1373 & 25146 & 696 & 2706 & 323 & 4805 & 219 & 2499 & 234 & 3939 & 155 \\
\hline $\operatorname{csv}-2$ & 13751 & 933 & 16909 & 903 & 2718 & 253 & 3767 & 302 & 2897 & 187 & 3527 & 199 \\
\hline $\mathrm{csv}-3$ & 11979 & 760 & 13713 & 1017 & 3240 & 250 & 3911 & 299 & 2834 & 173 & 2897 & 209 \\
\hline esc- 1 & 5840 & 415 & 7894 & 239 & 1099 & 117 & 1586 & 115 & 1022 & 77 & 1229 & 95 \\
\hline esc -2 & 7174 & 517 & 9983 & 228 & 1100 & 124 & 1578 & 114 & 1012 & 77 & 1286 & 101 \\
\hline esc-3 & 5808 & 391 & 7196 & 309 & 1070 & 97 & 1341 & 134 & 1332 & 76 & 1673 & 106 \\
\hline esc- 4 & 5387 & 284 & 7185 & 337 & 1178 & 90 & 1636 & 137 & 824 & 67 & 1049 & 119 \\
\hline esc -5 & 6163 & 458 & 8189 & 216 & 1109 & 127 & 1465 & 110 & 1001 & 102 & 1467 & 85 \\
\hline net-1 & 12576 & 814 & 15727 & 877 & 2780 & 397 & 3652 & 448 & 2630 & 234 & 3406 & 278 \\
\hline net-2 & 12288 & 781 & 15861 & 905 & 2779 & 393 & 3796 & 453 & 2569 & 233 & 3367 & 285 \\
\hline net-3 & 13115 & 901 & 16672 & 844 & 2373 & 396 & 3199 & 443 & 2272 & 231 & 2940 & 291 \\
\hline net-4 & 12911 & 1806 & 17933 & 842 & 2446 & 455 & 3854 & 368 & 2157 & 281 & 3035 & 231 \\
\hline net-5 & 12004 & 739 & 14736 & 925 & 2360 & 379 & 3014 & 463 & 2796 & 237 & 3522 & 308 \\
\hline prttok & 2926 & 726 & 882 & 212 & 693 & 206 & 302 & 114 & 657 & 176 & 197 & 108 \\
\hline prttok2 & 3220 & 570 & 596 & 223 & 558 & 126 & 69 & 59 & 565 & 132 & 114 & 54 \\
\hline replace & 6208 & 1254 & 1539 & 157 & 1219 & 392 & 373 & 92 & 839 & 230 & 210 & 61 \\
\hline sched & 3186 & 589 & 208 & 93 & 459 & 136 & 34 & 32 & 743 & 216 & 81 & 34 \\
\hline sched2 & 1661 & 372 & 252 & 86 & 445 & 103 & 79 & 42 & 453 & 113 & 69 & 55 \\
\hline totinfo & 1883 & 261 & 54 & 98 & 415 & 93 & 11 & 48 & 392 & 66 & 10 & 38 \\
\hline
\end{tabular}

Table 3: Comparison of the number of compilations (C), executions (E), execution time (T, sec), and deleted lines (D) for W-ORBS, VSM-ORBS, and LDA-ORBS

ORBS-M with $\Gamma$ increasing and decreasing requires $48 \%$ and $50 \%$ more compilations than LDA-ORBS while deleting only $1.7 \%$ and $1.1 \%$ more lines than LDA-ORBS. The results imply that the different thresholds have minimal impact in terms of the lines that can be deleted using our lexical dependence approximation. Even so, we continue to consider different thresholds with the lexical deletion operators since their efficiency might differ from each other.

In summary for RQ1, while lexical deletion operators delete fewer lines, they use significantly fewer compilations and executions, reducing wall clock time. Both lexical deletion operators are highly attractive in terms of their per-deleted-line efficiency, motivating MOBS's use of multiple deletion operators.

\subsection{Operator Comparison}

To answer RQ2, we investigate the relative applicability of different deletion operators. This is done by applying each deletion operator to all non-comment lines of code in the program's source to identify which lines are successful application points. Let $W, V$, and $L$ be the set of lines against which $\operatorname{Dw}^{\delta}(\delta \in\{1, \ldots, 4\})$, and $\operatorname{Dvsm}^{\gamma}$ 


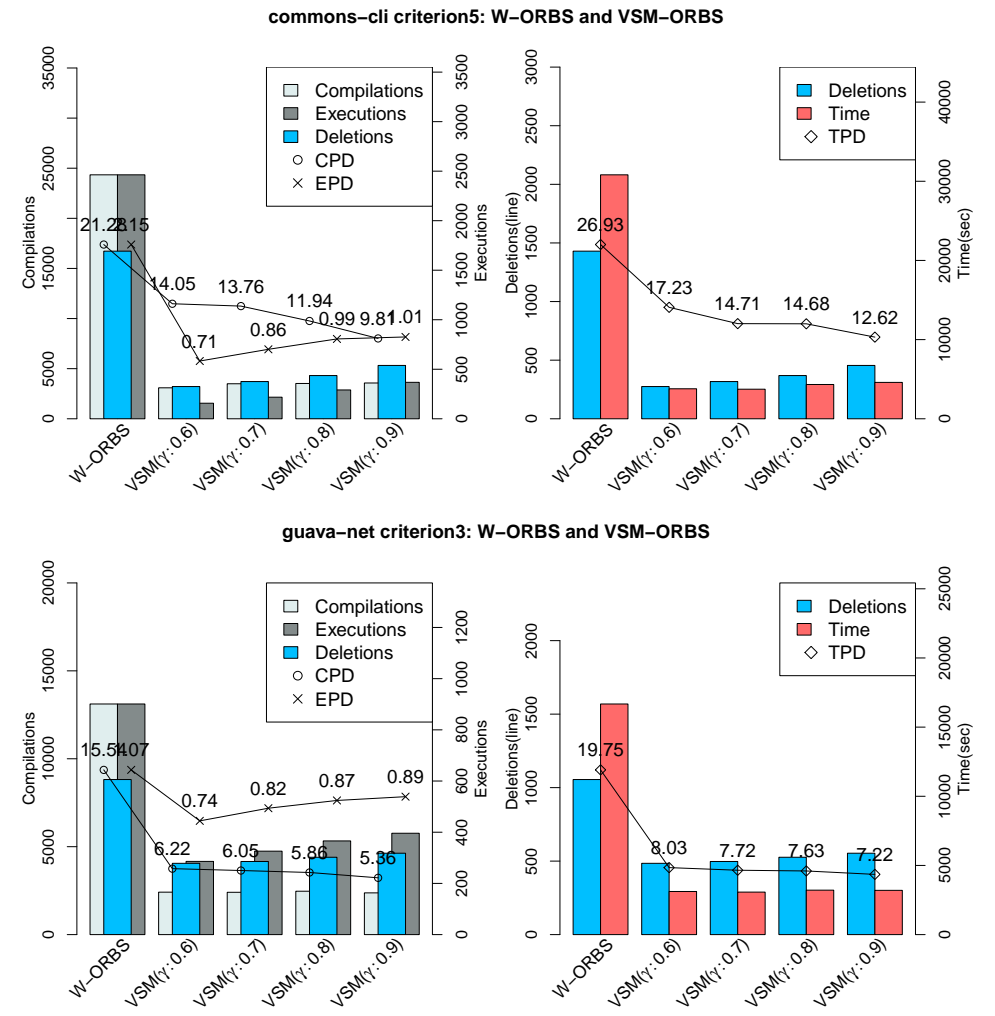

Figure 3: VSM-ORBS: Threshold analysis

$\operatorname{DLDA}^{\gamma}(\gamma \in\{0.6,0.7,0.8,0.9\})$ can be successfully applied: let $\sqcup$ denote the union of $W, V$, and $L$. In this experiment, we compute all pair-wise set differences to check how uniquely the operators can be applied to different locations.

Table 4 presents sizes of $W, V$, and $L$ as well as set differences between them, along with $D P S_{W}, D P S_{V}$, and $D P S_{L} . D P S_{W}$ is the average number of deleted lines across all successful applications of each $\mathrm{Dw}^{\delta}$ operator to all source code lines. Similarly, $D P S_{V}$ and $D P S_{L}$ are the average number of deleted lines across the applications of Dvsm and DLDA to all lines of source code, respectively.

In all cases, the size of $W$ is either identical or very close to that of the union, while sizes of $V$ and $L$ are significantly smaller than that of $W ;|V \backslash W|$ and $|L \backslash W|$ are always close to zero. Overall, this suggests that Dw can be applied to the largest number of lines successfully.

However, the results from the DPS analysis provide evidence that lexical deletion can improve the efficiency of ORBS. The results show that, for most of the Java subjects, $D P S_{V}$ is notably higher than $D P S_{W}$, suggesting that, when successful, Dvsm is capable of deleting more lines per attempt than Dw. $D P S_{L}$ shows mixed results. For C projects, $D P S$ values are almost equal between window and lexical deletion operators. We 


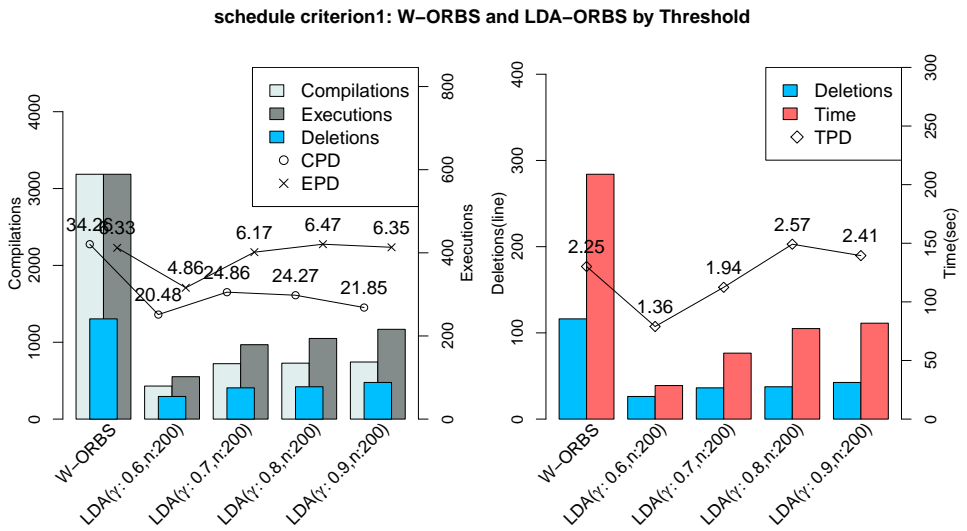

Figure 4: LDA-ORBS: Threshold value comparison

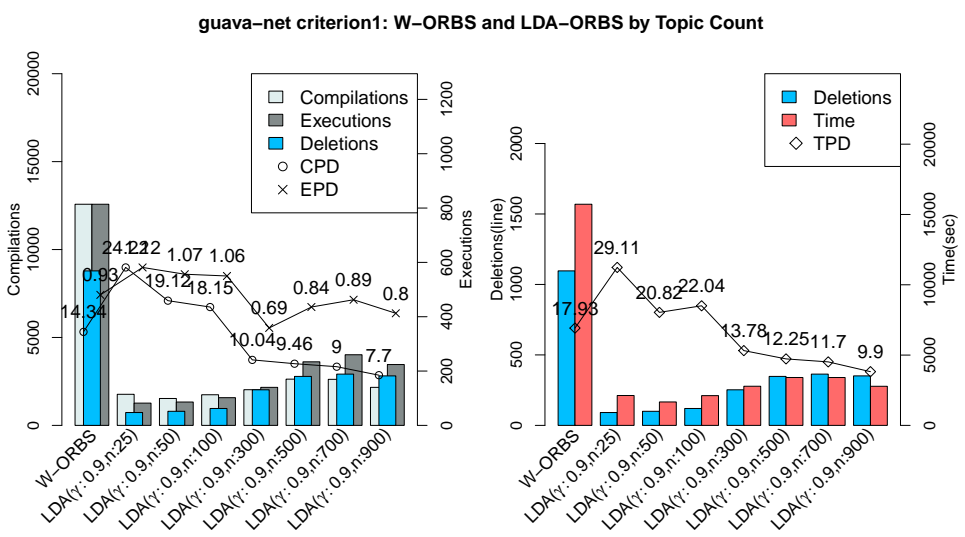

totinfo criterion1: W-ORBS and LDA-ORBS by Topic Count

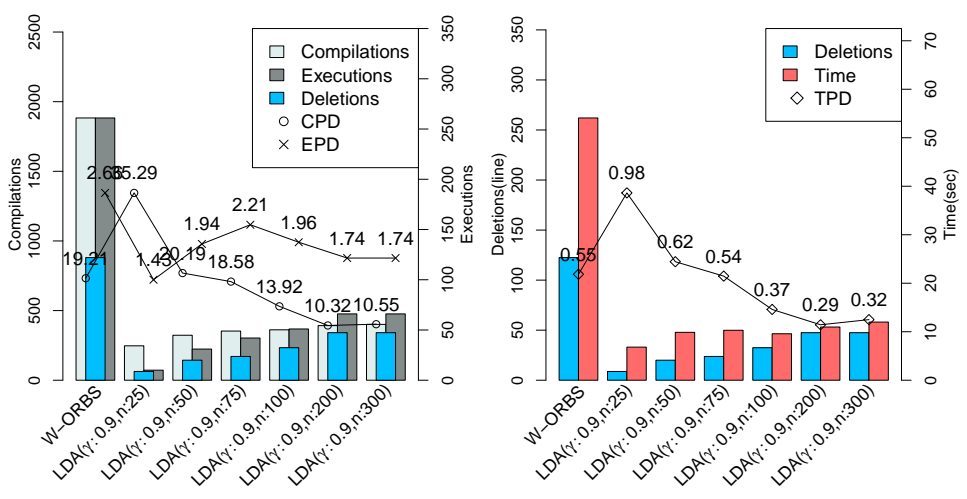

Figure 5: LDA-ORBS: Impact of topic count 


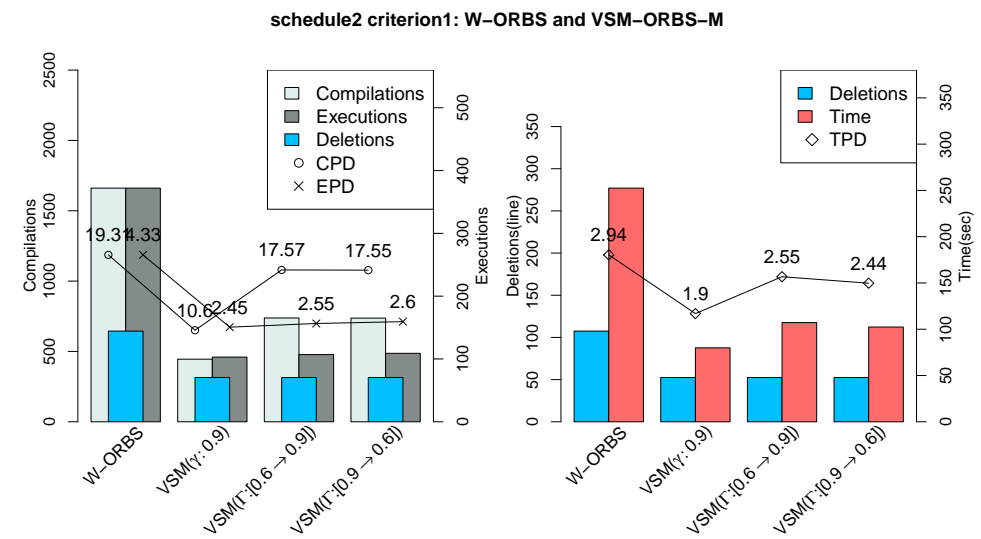

Figure 6: Efficiency of VSM-ORBS-M. The notation $\Gamma=[0.6 \rightarrow 0.9]$ denotes the values $0.6,0.7,0.8,0.9$, while $[0.9 \rightarrow 0.6]$ denotes the same values in decreasing order.

suspect that, due to the smaller size of these subjects, there are fewer similar source code lines for the lexical operators to exploit.

Note that higher DPS values do not necessarily mean that the corresponding operator will be highly applicable, as it measures the expected deletions per successful application. However, the results in Table 4 suggest that, if applied appropriately, lexical deletion operators stand to improve the efficiency of ORBS by deleting a greater number of lines per attempt.

The contrast in operator behaviours provides an answer to $\mathbf{R Q 2}$ and suggests the use of both window and lexical deletion operators during observation-based slicing. We initially tried a naive combination that ran W-ORBS on the slice generated by VSM-ORBS or LDA-ORBS. The strategy first deletes lines that can be detected using lexical deletion and then applies window deletion to the remaining lines. In doing so, it aims to combine the efficiency gain of lexical deletion with the smaller slices attained using window deletion.

Figure 7 compares the results of applying VSM-ORBS or LDA-ORBS followed by W-ORBS to those attained by applying W-ORBS alone. For the numerator, the number of iterations, compilations, executions, lines deleted, and the time taken is the sum of the value for VSM-ORBS or LDA-ORBS plus that for W-ORBS. The box plots in the figure summarize the ratios for each of the 24 slicing criteria studied. The green box is the ratio of W-ORBS after VSM-ORBS to W-ORBS, while the yellow box is the ratio of W-ORBS after LDA-ORBS to W-ORBS. On average, there is a slight increase in the number of deleted lines. However, the cost (e.g., the number of compilations, executions, and the time taken) increase dramatically. Interestingly, the initial application of lexical deletion does reduce the number iterations used in the subsequent application of W-ORBS. In 11 of the 24 slicing criteria, W-ORBS following VSM-ORBS or LDA-ORBS requires one fewer iteration, while for replace does it required two fewer.

The data shows that applying window deletion as a "second pass" does not improve slicing efficiency. Even so, initial lexical deletions do help to break certain dependence 


\begin{tabular}{|c|c|c|c|c|c|c|c|c|c|c|c|c|c|}
\hline \multirow{2}{*}{ Criteria } & \multirow{2}{*}{$\sqcup$} & \multirow{2}{*}{$W$} & \multirow{2}{*}{$V$} & \multirow{2}{*}{$L$} & \multirow{2}{*}{$W \backslash V$} & \multirow{2}{*}{$W \backslash L$} & \multirow{2}{*}{$V \backslash W$} & \multirow{2}{*}{$V \backslash L$} & \multirow{2}{*}{$L \backslash W$} & \multirow{2}{*}{$L \backslash V$} & \multicolumn{3}{|c|}{$D P S$} \\
\hline & & & & & & & & & & & $W$ & $V$ & $L$ \\
\hline cli-1 & 661 & 660 & 288 & 213 & 373 & 447 & 1 & 102 & 0 & 27 & 2.11 & 3.98 & 1.22 \\
\hline cli-2 & 804 & 802 & 347 & 285 & 456 & 518 & 1 & 95 & 1 & 33 & 2.11 & 4.45 & 1.94 \\
\hline cli-3 & 767 & 766 & 363 & 280 & 404 & 486 & 1 & 108 & 0 & 25 & 2.15 & 4.37 & 2.0 \\
\hline cli-4 & 549 & 548 & 225 & 192 & 323 & 357 & 0 & 58 & 1 & 25 & 2.04 & 5.35 & 2.84 \\
\hline cli-5 & 722 & 721 & 334 & 263 & 388 & 458 & 1 & 96 & 0 & 25 & 2.13 & 4.26 & 1.96 \\
\hline CSV-1 & 530 & 530 & 197 & 141 & 333 & 389 & 0 & 67 & 0 & 11 & 2.31 & 3.76 & 1.59 \\
\hline csv-2 & 624 & 623 & 254 & 176 & 369 & 448 & 0 & 89 & 1 & 11 & 2.31 & 3.48 & 1.62 \\
\hline $\operatorname{csv}-3$ & 670 & 670 & 255 & 186 & 415 & 484 & 0 & 82 & 0 & 13 & 2.34 & 3.46 & 1.55 \\
\hline esc-1 & 190 & 185 & 95 & 80 & 92 & 109 & 2 & 29 & 4 & 14 & 1.96 & 5.48 & 2.03 \\
\hline esc -2 & 169 & 160 & 89 & 77 & 73 & 91 & 2 & 25 & 8 & 13 & 1.89 & 5.75 & 2.04 \\
\hline esc-3 & 215 & 207 & 111 & 89 & 98 & 125 & 2 & 38 & 7 & 16 & 1.98 & 5.08 & 1.85 \\
\hline esc- 4 & 252 & 237 & 118 & 111 & 122 & 140 & 3 & 30 & 14 & 23 & 2.03 & 4.78 & 4.93 \\
\hline esc -5 & 176 & 170 & 90 & 73 & 82 & 102 & 2 & 28 & 5 & 11 & 1.94 & 5.66 & 2.15 \\
\hline net-1 & 679 & 675 & 421 & 269 & 257 & 409 & 3 & 162 & 3 & 10 & 2.31 & 2.76 & 1.76 \\
\hline net-2 & 667 & 658 & 422 & 275 & 239 & 391 & 3 & 164 & 8 & 17 & 2.3 & 2.78 & 1.62 \\
\hline net-3 & 649 & 642 & 416 & 288 & 228 & 361 & 2 & 147 & 7 & 19 & 2.32 & 2.8 & 2.53 \\
\hline net -4 & 606 & 602 & 341 & 222 & 265 & 382 & 4 & 130 & 2 & 11 & 2.28 & 3.05 & 1.82 \\
\hline net-5 & 702 & 697 & 438 & 292 & 262 & 408 & 3 & 161 & 3 & 15 & 2.31 & 2.73 & 1.79 \\
\hline prttok & 179 & 179 & 60 & 56 & 119 & 123 & 0 & 10 & 0 & 6 & 2.25 & 1.38 & 1.26 \\
\hline prttok2 & 189 & 184 & 107 & 95 & 82 & 93 & 5 & 24 & 4 & 12 & 2.23 & 2.81 & 3.21 \\
\hline replace & 120 & 120 & 75 & 51 & 45 & 69 & 0 & 26 & 0 & 2 & 1.42 & 1.36 & 1.32 \\
\hline sched & 91 & 90 & 40 & 52 & 51 & 39 & 1 & 2 & 1 & 14 & 1.61 & 1.39 & 1.45 \\
\hline sched2 & 54 & 54 & 30 & 26 & 24 & 28 & 0 & 6 & 0 & 2 & 1.56 & 1.15 & 1.34 \\
\hline totinfo & 92 & 90 & 47 & 38 & 45 & 54 & 2 & 10 & 2 & 1 & 2.01 & 1.38 & 1.43 \\
\hline
\end{tabular}

Table 4: Comparison between deletion operators

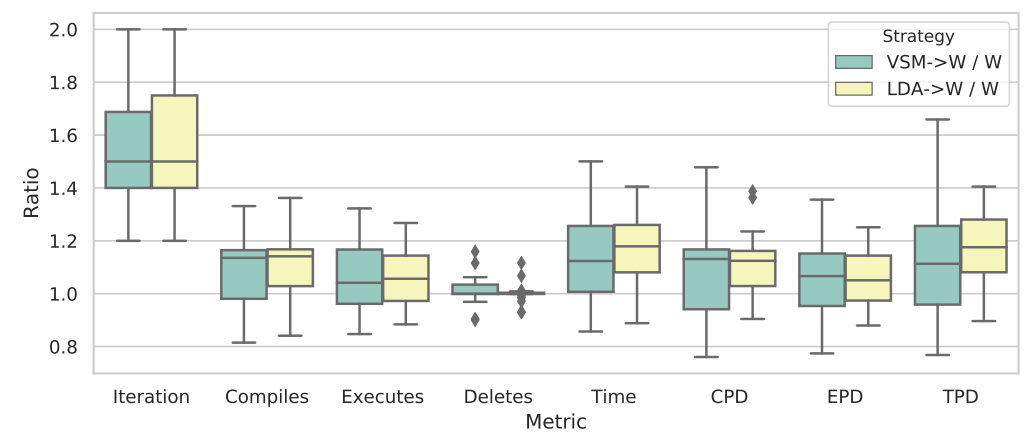

Figure 7: Ratios comparing VSM-ORBS or LDA-ORBS followed by W-ORBS to W-ORBS alone.

chains and thus reduce the slice size and the number of iterations W-ORBS requires. This potential synergy between window and lexical deletion further motivates MOBS. The interplay between the two suggests that a more sophisticated combination might be able to exploit the strengths of each approach. It is this potential that motivates our introduction and subsequent study of MOBS. 


\begin{tabular}{|c|c|c|c|c|c|c|c|c|c|c|c|c|}
\hline \multirow{2}{*}{ Criteria } & \multicolumn{4}{|c|}{ Dw with $\delta=$} & \multicolumn{4}{|c|}{ Dvsm with $\gamma=$} & \multicolumn{4}{|c|}{ DLDA with $\gamma=$} \\
\hline & 1 & 2 & 3 & 4 & 0.6 & 0.7 & 0.8 & 0.9 & 0.6 & 0.7 & 0.8 & 0.9 \\
\hline cli-1 & 0.162 & 0.069 & 0.082 & 0.059 & 0.065 & 0.080 & 0.098 & 0.119 & 0.050 & 0.058 & 0.072 & 0.088 \\
\hline cli-2 & 0.155 & 0.062 & 0.088 & 0.052 & .064 & 0.080 & 0.097 & 0.118 & 0.055 & 0.063 & 0.076 & 0.091 \\
\hline cli-3 & 0.153 & 0.073 & 0.081 & 0.063 & 0.061 & 0.077 & 0.096 & 0.119 & 0.050 & 0.059 & 0.076 & 0.092 \\
\hline cli-4 & 0.159 & 0.056 & 0.075 & 0.048 & 0.064 & 0.082 & 0.103 & 0.113 & 0.054 & 0.066 & 0.085 & 0.095 \\
\hline cli-5 & 0.152 & 0.074 & 0.077 & 0.061 & 0.062 & 0.077 & 0.092 & 0.114 & 0.056 & 0.065 & 0.079 & 0.091 \\
\hline csv-1 & 0.139 & 0.075 & 0.098 & 0.081 & 0.059 & 0.077 & 0.094 & 0.109 & 0.059 & 0.060 & 0.069 & 0.078 \\
\hline $\mathrm{csv}-2$ & 0.135 & 0.080 & 0.102 & 0.079 & 0.061 & 0.081 & 0.098 & 0.111 & 0.054 & 0.057 & 0.068 & 0.075 \\
\hline $\mathrm{csv}-3$ & 0.131 & 0.076 & 0.110 & 0.079 & 0.060 & 0.079 & 0.096 & 0.108 & 0.057 & 0.060 & 0.069 & 0.077 \\
\hline esc- 1 & 0.140 & 0.043 & 0.049 & 0.040 & 0.088 & 0.098 & 0.107 & 0.114 & 0.068 & 0.071 & 0.089 & 0.094 \\
\hline esc- 2 & 0.142 & 0.045 & 0.042 & 0.036 & 0.092 & 0.104 & 0.109 & 0.116 & 0.060 & 0.067 & 0.089 & 0.097 \\
\hline esc-3 & 0.146 & 0.046 & 0.052 & 0.043 & 0.093 & 0.103 & 0.113 & 0.126 & 0.059 & 0.059 & 0.074 & 0.087 \\
\hline esc- 4 & 0.131 & 0.041 & 0.052 & 0.043 & 0.084 & 0.092 & 0.101 & 0.111 & 0.074 & 0.073 & 0.095 & 0.103 \\
\hline esc -5 & 0.145 & 0.047 & 0.048 & 0.040 & 0.093 & 0.104 & 0.112 & 0.118 & 0.056 & 0.062 & 0.086 & 0.088 \\
\hline net-1 & 118 & 0.072 & 0.080 & 0.073 & 0.096 & 0.101 & 0.108 & 0.112 & 0.049 & 0.053 & 0.064 & 0.074 \\
\hline net-2 & 0.120 & 0.074 & 0.079 & 0.071 & 0.098 & 0.104 & 0.111 & 0.115 & 0.044 & 0.048 & 0.063 & 0.073 \\
\hline net-3 & 0.115 & 0.071 & 0.075 & 0.072 & 0.095 & 0.100 & 0.107 & 0.111 & 0.050 & 0.056 & 0.069 & 0.079 \\
\hline net-4 & 0.125 & 0.063 & 0.078 & 0.070 & 0.094 & 0.101 & 0.111 & 0.116 & 0.048 & 0.053 & 0.067 & 0.075 \\
\hline net-5 & 0.117 & 0.069 & 0.076 & 0.072 & 0.095 & 0.101 & 0.108 & 0.112 & 0.051 & 0.056 & 0.068 & 0.074 \\
\hline prttok & 0.134 & 0.091 & 0.080 & 0.073 & 0.065 & 0.077 & 0.089 & 0.092 & 0.073 & 0.073 & 0.075 & 0.080 \\
\hline prttok2 & 0.160 & 0.099 & 0.130 & 0.082 & 0.052 & 0.055 & 0.069 & 0.085 & 0.058 & 0.060 & 0.067 & 0.084 \\
\hline replace & 0.191 & 0.047 & 0.017 & 0.015 & 0.069 & 0.073 & 0.109 & 0.140 & 0.075 & 0.075 & 0.094 & 0.096 \\
\hline sched & 0.180 & 0.051 & 0.035 & 0.012 & 0.055 & 0.090 & 0.094 & 0.118 & 0.082 & 0.090 & 0.090 & 0.102 \\
\hline sched2 & 0.151 & 0.034 & 0.041 & 0.009 & 0.073 & 0.084 & 0.089 & 0.089 & 0.096 & 0.103 & 0.114 & 0.116 \\
\hline totinfo & 0.137 & 0.054 & 0.041 & 0.056 & 0.090 & 0.092 & 0.099 & 0.106 & 0.083 & 0.079 & 0.081 & 0.081 \\
\hline
\end{tabular}

Table 5: The selection probability for FOS with applicability for each deletion operator

\subsection{Operator Selection Strategy}

Tables 5 and 6 contain the probability of each operators calculated using applicability and effect for FOS, respectively (Section 4.2.2). Table 5 shows that operators that delete fewer lines tend to take higher probability (i.e., opportunities to delete a large number of lines together are rare) for all subjects. $\mathrm{Dw}^{1}$ has the highest probability, and consequently will be most frequently selected by FOS-MOBS when using applicability. However, in an interesting contrast, Table 6 shows that Dvsm has a much higher probability than other deletion operators for the Java projects (i.e., when those rare opportunities arise, Dvsm can delete a sufficiently large number of lines to overcome its rareness). Among the Dw operators, applicability shows a negative correlation while a positive correlation is observed between $\delta$ and effect. Note that there does not exist an observable trend in effect for the $\mathrm{C}$ subjects. We suspect the higher verbosity of Java code compared to C may yield the higher applicability of Dvsm, due to the richer lexical information in the source code: however, further study is required to confirm this. Finally, probabilities of DLDA remain relatively low across all subjects.

Figure 8 shows the result of Vargha-Delaney $\hat{A}_{12}$ statistic (Vargha and Delaney, 2000) for the TPD values of the four different operator selection strategies used by MOBS. Each of ROS, APP, EFF, and UNI appearing in the name of columns represents MOBS with rolling operator selection, fixed operator selection using 'applicability', 'effect', and uniform proportion, respectively. To facilitate the comparison to W-ORBS, we terminate MOBS after the number of iterations W-ORBS requires to complete a slice, and compute TPD values until that iteration. In column $P: Q$, the $\hat{A}_{12}$ statistic 


\begin{tabular}{|c|c|c|c|c|c|c|c|c|c|c|c|c|}
\hline \multirow{2}{*}{ Criteria } & \multicolumn{4}{|c|}{ Dw with $\delta=$} & \multicolumn{4}{|c|}{ Dvsm with $\gamma=$} & \multicolumn{4}{|c|}{ DLDA with $\gamma=$} \\
\hline & 1 & 2 & 3 & 4 & 0.6 & 0.7 & 0.8 & 0.9 & 0.6 & 0.7 & 0.8 & 0.9 \\
\hline cli-1 & 0.064 & 0.054 & 0.097 & 0.093 & 0.140 & 0.138 & 0.134 & 0.152 & 0.024 & 0.029 & 0.035 & 0.040 \\
\hline cli-2 & 0.053 & 0.042 & 0.091 & 0.072 & 131 & 0.148 & 0.133 & 0.137 & 0.046 & 0.046 & 0.051 & 0.048 \\
\hline cli-3 & 0.053 & 0.050 & 0.084 & 0.087 & 0.126 & 0.142 & 0.131 & 0.135 & 0.043 & 0.045 & 0.053 & 0.051 \\
\hline cli-4 & 0.046 & 0.032 & 0.065 & 0.055 & 0.132 & 0.155 & 0.142 & 0.129 & 0.035 & 0.062 & 0.087 & 0.062 \\
\hline cli-5 & 0.054 & 0.053 & 0.082 & 0.087 & 0.121 & 0.143 & 0.129 & 0.128 & 0.046 & 0.053 & 0.055 & 0.049 \\
\hline csv-1 & 0.053 & 0.058 & 0.112 & 0.124 & 0.113 & 0.121 & 0.124 & 0.132 & 0.039 & 0.040 & 0.040 & 0.044 \\
\hline $\mathrm{csv}-2$ & 0.053 & 0.063 & 0.120 & 0.124 & 0.118 & 0.120 & 0.118 & 0.122 & 0.037 & 0.041 & 0.042 & 0.041 \\
\hline $\mathrm{csv}-3$ & 0.052 & 0.060 & 0.131 & 0.125 & 0.116 & 0.118 & 0.117 & 0.120 & 0.039 & 0.038 & 0.041 & 0.043 \\
\hline esc- 1 & 0.041 & 0.025 & 0.043 & 0.047 & .159 & 0.163 & 0.165 & 0.165 & 0.047 & 0.046 & 0.050 & 0.048 \\
\hline esc- 2 & 0.040 & 0.025 & 0.036 & 0.040 & 0.167 & 0.172 & 0.170 & 0.171 & 0.043 & 0.046 & 0.044 & 0.046 \\
\hline esc-3 & 0.044 & 0.028 & 0.047 & 0.053 & 0.164 & 0.168 & 0.169 & 0.171 & 0.041 & 0.039 & 0.040 & 0.037 \\
\hline esc- 4 & 0.032 & 0.020 & 0.038 & 0.042 & 0.113 & 0.113 & 0.114 & 0.114 & 0.104 & 0.103 & 0.108 & 0.100 \\
\hline esc -5 & 0.040 & 0.026 & 0.040 & 0.044 & 0.165 & 0.169 & 0.170 & 0.170 & 0.046 & 0.047 & 0.045 & 0.037 \\
\hline net-1 & 050 & 0.061 & 0.101 & 123 & 135 & 0.122 & 0.117 & 0.113 & 0.040 & 0.041 & 0.044 & 0.053 \\
\hline net- 2 & 0.051 & 0.063 & 0.101 & 0.122 & 0.139 & 0.127 & 0.123 & 0.117 & 0.032 & 0.033 & 0.044 & 0.048 \\
\hline net-3 & 0.045 & 0.055 & 0.088 & 0.112 & 0.123 & 0.113 & 0.110 & 0.105 & 0.049 & 0.059 & 0.069 & 0.074 \\
\hline net- 4 & 0.050 & 0.050 & 0.094 & 0.112 & 0.141 & 0.128 & 0.126 & 0.121 & 0.035 & 0.040 & 0.049 & 0.053 \\
\hline net-5 & 0.050 & 0.059 & 0.097 & 0.123 & 0.131 & 0.121 & 0.118 & 0.113 & 0.045 & 0.044 & 0.050 & 0.050 \\
\hline prttok & 0.050 & 0.067 & 0.087 & 0.106 & 0.101 & 0.085 & 0.074 & 0.074 & 0.093 & 0.092 & 0.088 & 0.082 \\
\hline prttok2 & 0.091 & 0.111 & 0.219 & 0.182 & 0.053 & 0.043 & 0.051 & 0.057 & 0.042 & 0.042 & 0.048 & 0.061 \\
\hline replace & 0.140 & 0.067 & 0.034 & 0.040 & 0.074 & 0.073 & 0.107 & 0.136 & 0.076 & 0.076 & 0.088 & 0.089 \\
\hline sched & 0.135 & 0.076 & 0.079 & 0.035 & 0.047 & 0.079 & 0.082 & 0.100 & 0.085 & 0.091 & 0.091 & 0.100 \\
\hline sched2 & 0.102 & 0.047 & 0.084 & 0.025 & 0.075 & 0.085 & 0.082 & 0.076 & 0.102 & 0.102 & 0.115 & 0.104 \\
\hline totinfo & 0.087 & 0.067 & 0.074 & 0.138 & 0.083 & 0.084 & 0.086 & 0.087 & 0.081 & 0.070 & 0.071 & 0.071 \\
\hline
\end{tabular}

Table 6: The selection probability for FOS with effect for each deletion operator

is the probability that a score sampled at random from the first population, $P$, will be greater than a score sampled at random from the second, $Q$. Columns starting with ROS contain more dark blue cells than others, indicating that ROS tends to show higher efficiency (marked by lower TPD). Variants of FOS show little difference from each other. Based on these results, we answer $\mathbf{R Q 3}$ by concluding that the Rolling Operator Selection (ROS) has the best performance.

\subsection{Comparison between MOBS and W-ORBS}

We compare MOBS to W-ORBS. Table 7 shows the means and standard deviations for the wall clock execution time, the number of deleted lines, and per-deletion efficiency for W-ORBS and MOBS using the four different operator selection strategies. The largest number of deleted lines, the shortest execution time, and the lowest TPD values among the four strategies are typeset in bold. The box plots shown in Figure 9 show the distributions of these values at the end of each iteration for four slicing criteria. Note that the $y$-axis for the execution time box plots on the right use a logarithmic scale.

ROS-MOBS (found to be the most efficient variant in RQ3) deletes $63 \%$ to $83 \%$ of lines deleted by W-ORBS in $27 \%$ to $45 \%$ of the time required by W-ORBS. The worst case efficiency of ROS-MOBS is observed in sched, whose TPD value is highest when compared to that of W-ORBS. For this program ROS-MOBS only deletes $63 \%$ of the lines deleted by W-ORBS, while taking only $40 \%$ of its execution time. However, even in this worst case, the trade-off is better than linear. Based on these results, we answer RQ4 by concluding that ROS-MOBS can be both effective and efficient, being 


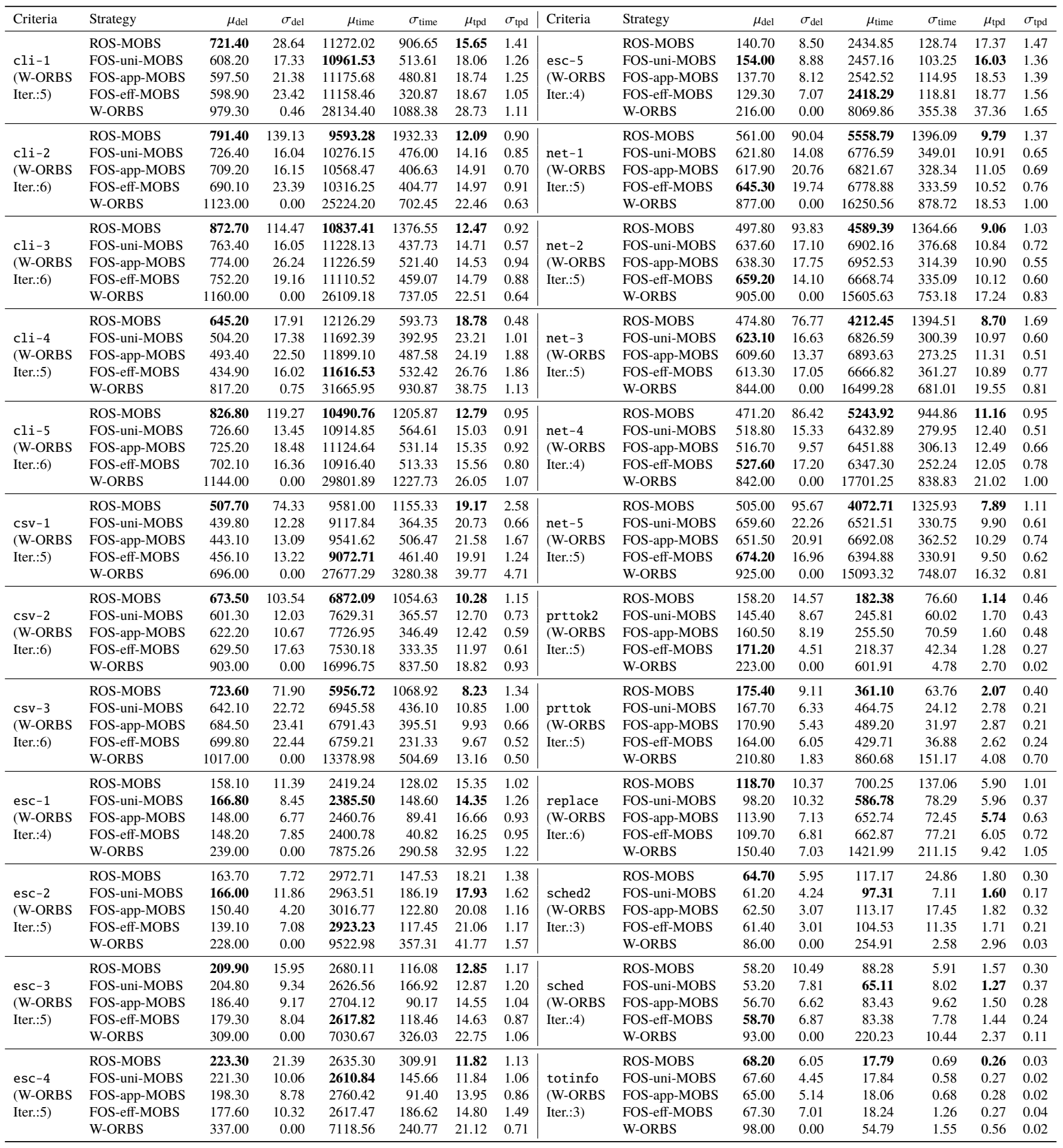

Table 7: Statistics on execution time and the number of deleted lines for W-ORBS and MOBS . 


\begin{tabular}{|c|c|c|c|c|c|c|c|}
\hline & ROS : UNI & ROS : APP & ROS : EFF & UNI : APP & UNI : EFF & APP : EFF & \multirow{25}{*}{$\hat{A}_{12}$} \\
\hline cli-1 - & 0.12 & 0.06 & 0.04 & 0.31 & 0.39 & 0.56 & \\
\hline cli-2 - & 0.05 & 0.03 & 0.03 & 0.2 & 0.23 & 0.44 & \\
\hline cli-3 - & 0.01 & 0.06 & 0.04 & 0.54 & 0.5 & 0.44 & \\
\hline cli-4 - & 0 & 0 & 0 & 0.37 & 0.03 & 0.15 & \\
\hline cli-5- & 0.04 & 0.01 & 0.01 & 0.38 & 0.32 & 0.39 & \\
\hline csv-1 - & 0.26 & 0.2 & 0.39 & 0.36 & 0.69 & 0.78 & \\
\hline csv-2 - & 0.06 & 0.06 & 0.11 & 0.61 & 0.77 & 0.67 & \\
\hline csv-3 - & 0.07 & 0.17 & 0.18 & 0.8 & 0.87 & 0.66 & \\
\hline esc-1 - & 0.72 & 0.18 & 0.26 & 0.06 & 0.13 & 0.62 & \\
\hline esc-2 - & 0.56 & 0.07 & 0.04 & 0.12 & 0.03 & 0.29 & \\
\hline esc-3 - & 0.47 & 0.14 & 0.11 & 0.16 & 0.13 & 0.48 & \\
\hline esc-4 - & 0.52 & 0.05 & 0.06 & 0.09 & 0.05 & 0.32 & \\
\hline esc-5 - & 0.75 & 0.27 & 0.23 & 0.1 & 0.09 & 0.45 & \\
\hline net-1 - & 0.27 & 0.22 & 0.3 & 0.44 & 0.71 & 0.74 & \\
\hline net-2 - & 0.1 & 0.07 & 0.23 & 0.49 & 0.75 & 0.84 & \\
\hline net-3 - & 0.17 & 0.17 & 0.18 & 0.3 & 0.47 & 0.66 & \\
\hline net-4 - & 0.12 & 0.15 & 0.21 & 0.41 & 0.7 & 0.69 & \\
\hline net-5 - & 0.09 & 0.03 & 0.12 & 0.36 & 0.68 & 0.83 & \\
\hline prttok - & 0.16 & 0.23 & 0.35 & 0.58 & 0.81 & 0.69 & \\
\hline prttok2 - & 0.03 & 0 & 0.14 & 0.41 & 0.65 & 0.75 & \\
\hline replace - & 0.58 & 0.64 & 0.55 & 0.59 & 0.5 & 0.47 & \\
\hline sched - & 0.71 & 0.49 & 0.62 & 0.32 & 0.34 & 0.58 & \\
\hline sched2 - & 0.78 & 0.56 & 0.64 & 0.23 & 0.25 & 0.54 & \\
\hline totinfo - & 0.46 & 0.31 & 0.42 & 0.33 & 0.46 & 0.57 & \\
\hline
\end{tabular}

Figure 8: Vargha-Delaney $\hat{A}_{12}$ on TPD between selection strategies. Each of ROS, APP, EFF, and UNI represents MOBS with rolling operator selection, fixed operator selection using 'applicability', 'effect', and uniform proportion, respectively.

capable of deleting an average of $69 \%$ of the number of lines deleted by W-ORBS, while requiring only $36 \%$ of wall clock execution time required by W-ORBS.

\subsection{Qualitative Analysis of the Slices}

This section answers RQ5 with a qualitative analysis of the slices. We investigate the differences between the slices generated by W-ORBS, VSM-ORBS, and MOBS. We omit LDA-ORBS from the analysis as the results hitherto clearly suggest it is not as effective as VSM-ORBS.

\subsubsection{Similarity between $W-O R B S$ and MOBS Slices}

Figure 10 shows our modified Jaccard similarity computed between the results of ROS-MOBS and W-ORBS for two slicing criteria, net-3 and replace. The values on the leftmost column represent the similarity between the slice produced by W-ORBS and ten trials of MOBS; values above the diagonal represent the similarity between the ten trials of MOBS where darker squares indicate greater similarity between the two slices. Except for small fluctuations, slices generated by MOBS are much more similar to each other, than they are similar to the slices generated by W-ORBS. This implies that, despite stochasticity, slices generated by MOBS share common patterns that are different from those generated by W-ORBS. We next consider whether lexical approximation of dependence is particularly effective or ineffective against specific types of statements. 


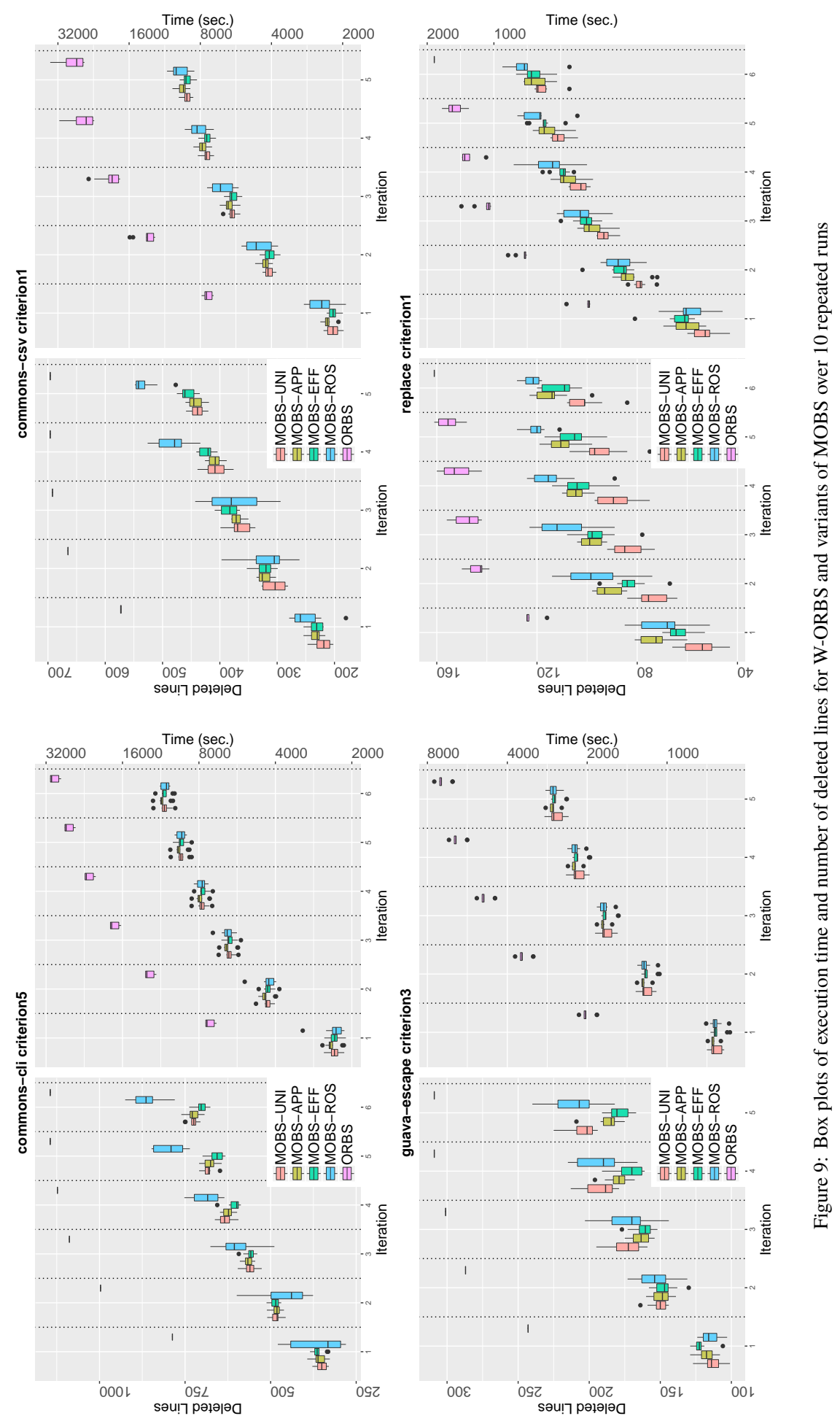



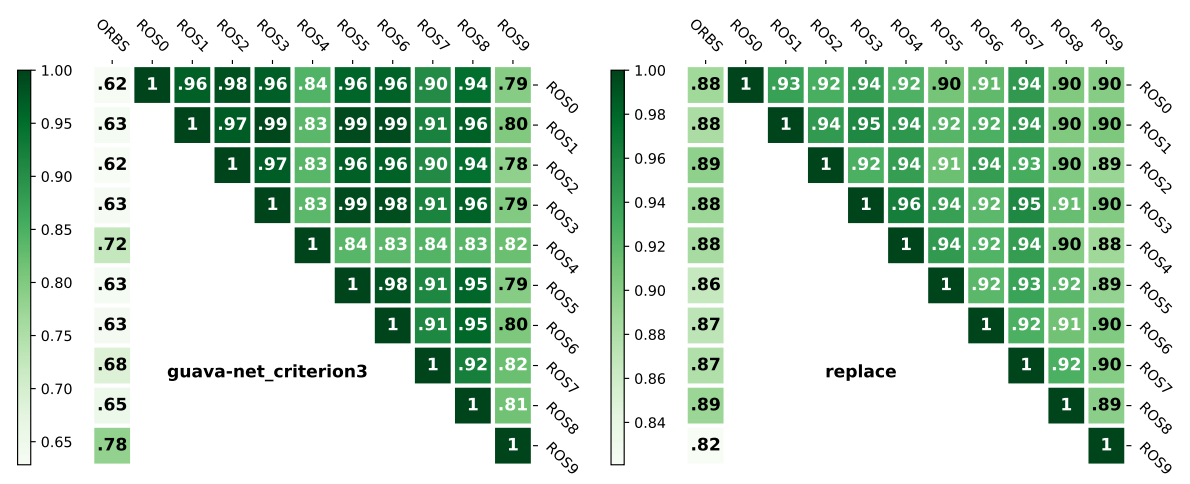

Figure 10: Jaccard similarity between ROS-MOBS and W-ORBS for net-3 and replace .

\subsubsection{Characteristics of Slices}

Table 8 shows the top thirty lines that are most frequently found in slices by VSMORBS but not in those by W-ORBS. We observe the following patterns that make it difficult for VSM-ORBS to delete these lines:

- Multi-line Statements: Lexical deletion operators do not try to delete consecutive lines. Thus, they may attempt to delete one line from a multi-line statement, raising compilation errors. This case includes lines ending with a left bracket that marks the beginning of a compound statement, such as if (it.hasNext ()) \{.

- Declarations: As can be seen in Section 7.1, the lexical deletion operators cannot delete as many lines as W-ORBS. Consequently, they often fail to delete all uses of a variable before attempting to delete its declaration. Similarly, they often fail to delete the body of a method before attempting to delete the method declarations. This means that declaration statements are harder for VSM-ORBS to delete than for W-ORBS.

- Frequent Lexemes: certain lexemes occur frequently throughout the code in many different, and potentially unrelated, semantic contexts. They may be lexically similar to each other, but this does not necessarily mean that there also exists dependence. For example, return this; is a lexeme that can be found in many different methods: attempting to delete all instances of return this; is most likely to fail, even if the specific instance under consideration can actually be deleted.

We investigate how capable W-ORBS, VSM-ORBS, and MOBS are at deleting lines having these characteristics. Figure 11 contains box plots that show how many lines of interest (i.e., either lines that are part of multi-line statements or declarations) can be deleted by each technique. Figure 12 shows the results of a similar analysis for frequent lexemes.

The box plots in Figure 11 show that, for all slicing criteria, VSM-ORBS deletes the fewest lines of interest, followed by MOBS, and W-ORBS, which deletes the most 


\begin{tabular}{l|r} 
Code line (Top thirty with frequency) & Freq. \\
\hline \} & 10228 \\
import static com.google.common.base.Preconditions.checkNotNull; & 212 \\
checkArgument ( & 168 \\
throw new IllegalArgumentException( & 144 \\
public String toString() \{ & 140 \\
return (TRUE); & 136 \\
currentOption = null; & 135 \\
eatTheRest = true; & 120 \\
return (this); & 104 \\
if (it.hasNext()) \{ & 92 \\
\{ & 92 \\
public int hashCode() \{ & 84 \\
import java.util.Iterator; & 80 \\
return (token); & 80 \\
hostPortString); & 76 \\
return (s); & 75 \\
return (false); & 73 \\
quote = true; & 72 \\
opt = Util.stripLeadingHyphens(opt); & 68 \\
return (true); & 64 \\
this.option = option; & 60 \\
import java.util.List; & 60 \\
if (opt == null) \{ & 60 \\
\}else if (matchingOpts.size() > 1) \{ & 60 \\
else\{ & 60 \\
import static com.google.common.base.Preconditions.checkArgument; ; \\
return (dest); & 60 \\
dest [3] = \%'; & 60 \\
import java.util.ArrayList; & 60 \\
import javax.annotation.Nullable; & 56 \\
\hline
\end{tabular}

Table 8: Lines that are retained in VSM-ORBS slices but not in W-ORBS slices.

lines of interest. The results suggest that syntactic structures in source code presents challenges to the lexical deletion operators, while the window deletion used by W-ORBS can circumvent this challenge.

Analysis of frequent lexemes requires a more subtle approach, as we cannot anticipate all such lexemes. Instead, we posit that these lexemes are more likely to consist of stop words, as our characterising definition of frequent lexemes is a lexeme that can appear in many different contexts. Non-stop words (i.e., solution-domain identifiers) are more likely to be bound to specific local contexts. Consequently, we compare the average number of non-stop word tokens per deleted line in Figure 12.

While there is variance in the difference, VSM-ORBS deletes more non-stop word tokens per deleted line than W-ORBS. One implication of this is that lexical deletion operators may find it more difficult to delete irrelevant control structures, as deleting them would require deleting lines with frequent lexemes that are related to control flow, such as return; or \} else \{. However, as long as lines that mostly consist of unique identifiers are deleted, the resulting slices may still be useful in cases where the user is interested more in understanding the dependencies between individual lines than in slicing out entire control flow structures. 

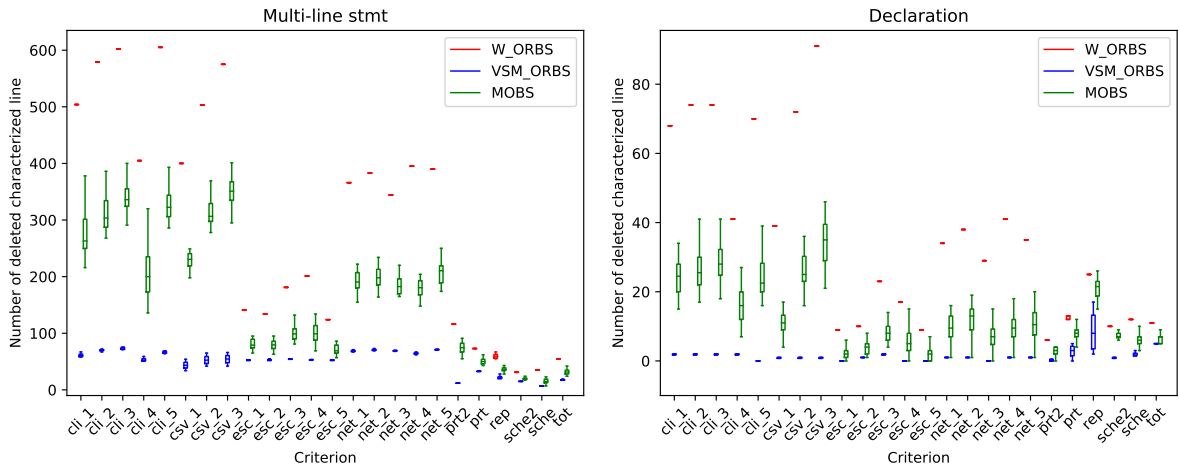

Figure 11: Number of deleted characterized lines

\subsection{Scalability}

To investigate how lexical dependency scales to both larger and multi-lingual systems, we consider the open-source project misaka ${ }^{6}$ as a benchmark program. Misaka is a CFFI-based binding for Hoedown, a fast markdown processing library written in C. The project consists of ten $\mathrm{C}$ files from Hoedown, which performs text parsing, and five Python files that wrap the $\mathrm{C}$ functions to produce a Python module. Misaka has a rich test suite containing 92 test cases written in Python that focus on evaluating the linkage between the Python and $\mathrm{C}$ functions, rather than the Hoedown library itself. The test suite consists of two unit tests that test the input arguments and 39 integration tests that test the binding of the $\mathrm{C}$ functions to Python methods. The remaining 41 system-level tests involve 41 different markdown text files and their corresponding HTML files.

We consider six slicing criteria for misaka, in an attempt to cover as diverse a set of functionalities as possible. We select slicing criteria in the $\mathrm{C}$ code, which are eventually reached from the Python test scripts through the CFFI binding. The first slicing criterion (crit-1) involves a variable tracking the index of the beginning of each line in a buffer while rendering a regular markdown document. The second slicing criterion (crit-2) targets the size of the text to render. The third slicing criterion (crit-3) is a variable containing the maximum size of the custom stack before it is changed by a method that grows the stack to a given size. The fourth slicing criterion (crit-4) targets the size of misaka's renderer object while allocating a regular HTML renderer. The fifth slicing criterion (crit-5) is a temporary variable which discriminates the starting index of the row from the padding when parsing a markdown table row. The last slicing criterion (crit-6) is the index of the beginning of a markdown link in the method that calculates the index of the end of the markdown link.

We ran W-ORBS, VSM-ORBS, and LDA-ORBS on the six slicing criteria using a threshold of 0.9 for $\gamma$ with both Dvsm and DLDA, and $n=500$ topics for DLDA. We also ran ROS-MOBS ten times for each slicing criteria. The results for VSM-ORBS and LDA-ORBS when compared to W-ORBS shows a similar trend to those of the previous

\footnotetext{
${ }^{6}$ https://misaka.61924.nl
} 


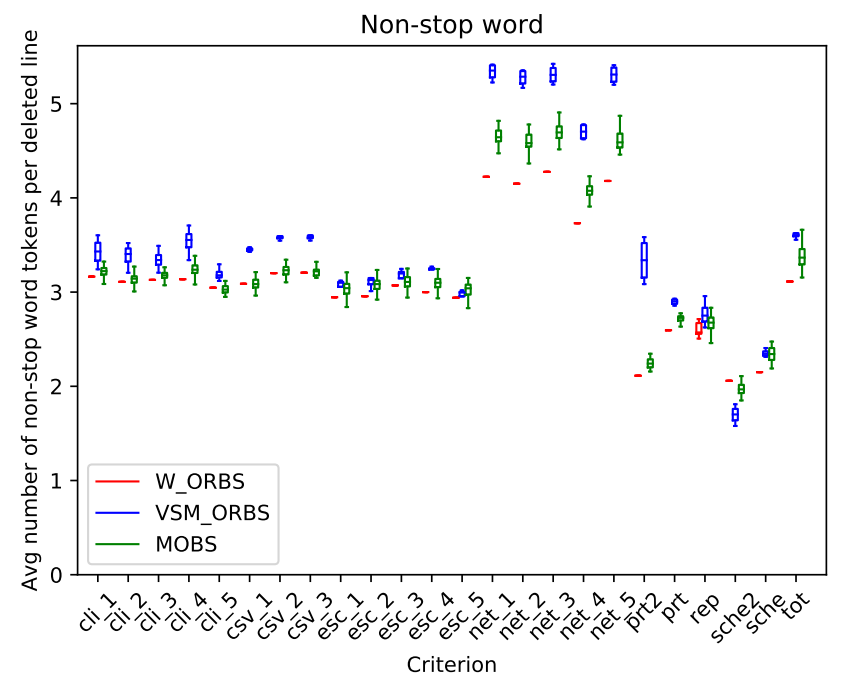

Figure 12: Average number of non-stop word tokens on a deleted line

experiments. On average for all slicing criterion, VSM-ORBS and LDA-ORBS slice the code 2.82 times and 2.31 times faster than W-ORBS while they delete $32.1 \%$ and $33.5 \%$ of lines that W-ORBS could delete, respectively.

Table 9 shows the result comparing W-ORBS and ROS-MOBS, and box plots in Figure 13 show the ratio between two. In Figure 13, the red box plot on the left represents how many times ROS-MOBS run faster compare to W-ORBS, and the blue box plot on the right represents the ratio of the number of deleted lines by ROS-MOBS to the number of deleted lines by W-ORBS. According to the result, crit-1 shows good performance from MOBS, where it deletes $71 \%$ of the lines deleted by W-ORBS while executing 2.6 times faster. MOBS shows poor performance on crit-4 and crit-5. For crit- 5 it is only 1.4 times faster while for crit- 4 the timing is essentially the same with the average speed up of 1.01 . For these slices it deletes $48 \%$ and $57 \%$ of the lines deleted by W-ORBS, respectively. We investigated this difference in performance. The main cause is the size of the slice. For slicing criterion crit-4, the size of the renderer object calculated by simply calling sizeof method on the object, has no control or data dependence between the surrounding source code. The dependency chains reaching crit4 are also simple and shallow, making most of the code easy to delete in early stages of W-ORBS and MOBS. The small number of remaining lines reduce MOBS's advantage of fewer deletion attempts on a single line when compared to W-ORBS. Similarly, the dependence of crit-5 is limited. It focuses solely on parsing the markdown table, which is a local function of the program, and has little dependence on other parsing methods in the program; thus, its slice size is the second smallest among all slicing criteria. On the other hand, criterion crit-1 targets the central logic of the Hoedown library. It occurs inside a while loop calculating the beginning index of every line of the document. Thus, the dependence chains weave through much of the code, and the final slice is the largest over all slicing criteria. A large number of remaining lines enhances the advantage of 


\begin{tabular}{l|l|rrrrr} 
Criterion & Strategy & Iteration & Compile & Execute & Deleted lines & Time \\
\hline \multirow{2}{*}{ crit-1 } & ROS-MOBS & 5.9 & 17,683 & 5,135 & 2,209 & 34,890 \\
& W-ORBS & 6.0 & 42,292 & 16,027 & 2,950 & 92,305 \\
\hline \multirow{2}{*}{ crit-2 } & ROS-MOBS & 5.1 & 13,561 & 2,937 & 2,145 & 22,765 \\
& W-ORBS & 6.0 & 25,612 & 8,849 & 3,880 & 50,970 \\
\hline \multirow{2}{*}{ crit-3 } & ROS-MOBS & 5.4 & 13,316 & 3,133 & 2,284 & 23,930 \\
& W-ORBS & 6.0 & 23,978 & 8,448 & 3,956 & 47,203 \\
\hline \multirow{2}{*}{ crit-4 } & ROS-MOBS & 4.9 & 10,579 & 1,602 & 2,626 & 10,953 \\
& W-ORBS & 6.0 & 12,700 & 2,787 & 4,642 & 10,683 \\
\hline \multirow{2}{*}{ crit-5 } & ROS-MOBS & 4.8 & 11,697 & 2,019 & 2,140 & 18,036 \\
& W-ORBS & 6.0 & 15,370 & 3,723 & 4,465 & 24,704 \\
\hline \multirow{2}{*}{ crit-6 } & ROS-MOBS & 5.4 & 16,231 & 3,994 & 2,488 & 30,922 \\
& W-ORBS & 6.0 & 26,878 & 9,163 & 3,794 & 50,762
\end{tabular}

Table 9: Statistics comparing ROS-MOBS and W-ORBS on the six misaka slicing criteria. The data for ROS-MOBS is an average of ten trial runs. Misaka has a total of 5,125 lines of code.

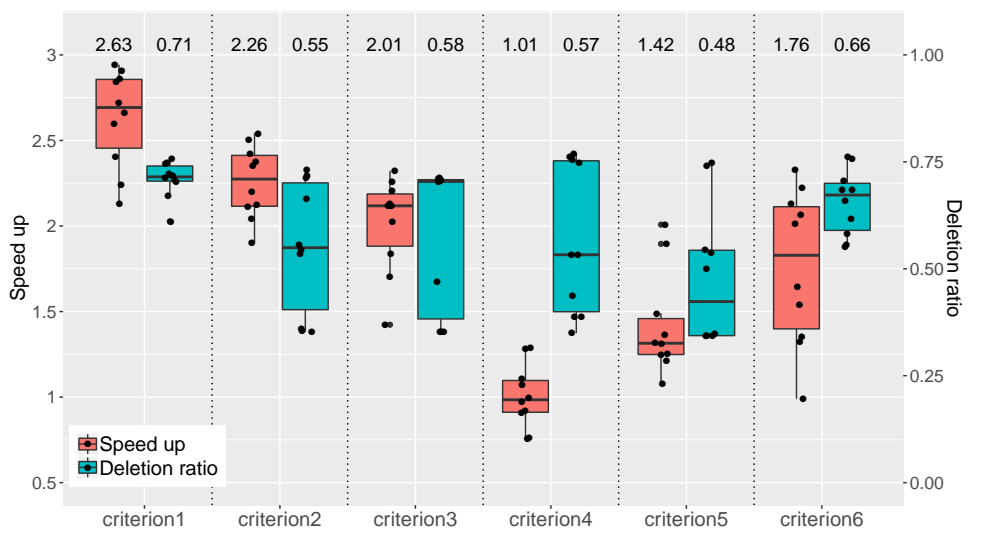

Figure 13: Ratios comparing the number of deleted lines and time taken by ROS-MOBS and W-ORBS

MOBS over W-ORBS, making the slice much faster than W-ORBS. The other three slicing criteria (crit-2, crit-3, and crit-6) show a similar trend. Their slices are smaller than the slice of crit-1, but larger than the slices of crit- 4 and crit-5. Both crit- 2 and crit- 6 involves greater dependence with other parsing methods than crit-5. Finally, slicing criterion crit-3 targets a function that increases the stack's size limit, which is used as a data structure to buffer parts of a document.

Table 10 shows examples of successful multi-lingual deletion by the lexical deletion operators. The first column shows which operator was used; both of Dvsm and DLDA are successfully applied to the code lines in the last row. Dvsm successfully deletes three lines: two from 'callbacks.py' and one from 'html.c'. Terms 'table', 'align', and 'left' are shared among the three causing them to be considered similar by the Vector Space Model. DLdA successfully delete lines from 'api.py', 'document.c', and 'html_smartypants.c', together. In this case, the terms 'hoedown', 'buffer', and 'text' are shared among three lines making them similar under LDA. Both Dvsm 


\begin{tabular}{c|l|l} 
Operator & File-name:Line-num & Code line \\
\hline \multirow{2}{*}{ Dvsm } & $\begin{array}{l}\text { callbacks.py:97 } \\
\text { callbacks.py:98 } \\
\text { hoedown/html.c:393 }\end{array}$ & $\begin{array}{l}\text { elif align_bit == TABLE_ALIGN_LEFT: } \\
\text { align = 'left, } \\
\text { case HOEDOWN_TABLE_ALIGN_LEFT: }\end{array}$ \\
\hline \multirow{2}{\text{DLDA}}{} & $\begin{array}{l}\text { api.py:29 } \\
\text { hoedown/document.c:2490 } \\
\text { hoedown/html_smartypants.c:195 }\end{array}$ & $\begin{array}{l}\text { lib.hoedown_buffer_puts(ib, text.encode('utf-8')) } \\
\text { hoedown_buffer_free(text); } \\
\text { hoedown_buffer_putc(ob, text [0]); }\end{array}$ \\
\hline $\begin{array}{c}\text { DvSM, } \\
\text { DLDA }\end{array}$ & $\begin{array}{l}\text { callbacks.py:125 } \\
\text { hoedown/html.c:635 }\end{array}$ & $\begin{array}{l}\text { result = renderer.blockhtml(text) } \\
\text { renderer->blockhtml = NULL; }\end{array}$
\end{tabular}

Table 10: Example successful multi-lingual deletions by the lexical deletion operators

and DLDA successfully deletes the lines result $=$ renderer. blockhtml (text) from 'callbacks.py' and renderer->blockhtml = NULL; from 'html.c', together. This result exemplifies that our new lexical deletion operators can capture (an approximation to) inter-language dependence in a multi-lingual program.

\subsection{Threats to Validity}

This section considers three threats to the validity of our experiments: external validity, internal validity, and construct validity. To begin with, external validity consider how well our results generalize to other environments. The subjects studied, shown in Table 1, include imperative and object-oriented codes of modest size. It is possible that our technique is not effective when larger programs or programs written in other languages are considered. Mitigating both of these threats is the previous application of ORBS to larger programs written in a range of programming languages. In fact this is one of ORBS strengths. The more serious external threat that larger programs bring is that the naming would become cluttered resulting in a lowering of the effectiveness of the IR based lexical approximations. In general, IR systems scale to very large corpora where then often perform better in the presence of more data.

Next, internal validity is the causal effect of the explanatory variables on the response variables. The use of ORBS, which empirically identifies the exact dynamic dependencies provides an excellent bellwether for assessing the lexical approximation of program dependence. When a lexical operator successful deletes a set of lexically related lines the ORBS's framework ensures that these lines are not semantically related to the slicing criterion and thus they can all be safely removed. In the other direction, just because one of a set of lexically similar lines can not be deleted, does not mean that other members of this set can not be deleted. Therefore we have strong evidence when a deletion is accepted, but the approximation is more suspect when a deletion is rejected.

The final threat considered is the threat to construct validity, which considers how well our approach measures what it claims. In our experiments construct validity is not a significant issue because we can directly measure dynamic dependence by running the program using the given test suite.

\section{Related Work}

Lexical analysis, especially techniques borrowed from Information Retrieval, have been widely studied and applied in software engineering. For example, LDA has 
been applied to program comprehension (Binkley et al., 2014b) and traceability recovery (Panichella et al., 2013); Vector Space Models have been applied to fault localisation, based on the intuition that bug reports and the faulty program code may tend to be lexically similar (Saha et al., 2013; Le et al., 2014, 2015; Wang et al., 2015). More broadly, application of Natural Language Processing (NLP) techniques to source code has been studied in the context of subjects such as the natural language model of source code (Hindle et al., 2012), coding conventions (Allamanis et al., 2014), and code snippet recommendation (Campbell and Treude, 2017). As far as we know, MOBS is the first approach to program slicing and, more generally, dependence analysis that exploits lexical information in program source code.

Since its introduction by Weiser in the 1970s (Weiser, 1979), program slicing has been widely studied and developed (Anderson and Teitelbaum, 2001; Horwitz et al., 1990; Amtoft and Banerjee, 2016; Hur et al., 2014). Static program slicing (Weiser, 1981) produces slices that are correct for all possible program executions, whereas dynamic slicing aims to tailor slices to a particular set of program inputs (Korel and Laski, 1988).

Many flavours of static slicing algorithms attempt to reduce the size of the resulting slice. Incremental Slicing (Orso et al., 2001) allows selection of the type of data dependencies that are considered while slicing. Stop-list slicing (Gallagher et al., 2006) allows the programmer to define variables that are not of interest, information that is subsequently used to purge the dependence graph before computing slices, resulting in smaller slices. Barrier Slicing (Krinke, 2003) allows the programmer to specify which parts of the program can and cannot be traversed while constructing the slice. A barrier is specified with a set of nodes or edges of the program's program dependence graph that cannot be passed during the graph traversal, also resulting in a focused and thus smaller slice.

Amorphous Slicing (Harman and Danicic, 1997) is an approach that aims to preserve the semantics of the program, but not its syntax. Amorphous slices use program transformation to simplify programs, preserving the semantics of the program with respect to the slicing criterion. In contrast MOBS (and ORBS) only transform a program using deletion.

Korel and Laski $(1988,1990)$ considered several algorithms to compute dynamic slices based on their definition. In contrast, most later work on dynamic slicing 'defines' dynamic slicing based on the algorithms used to compute it (e.g., Agrawal and Horgan (1990) and DeMillo et al. (1996)). Although many research prototypes and approaches exist (Beszedes et al., 2001, 2006; Mund and Mall, 2006; Szegedi and Gyimóthy, 2005; Zhang and Gupta, 2004; Zhang et al., 2007; Barpanda and Mohapatra, 2011), all these approaches are for a single specific programming language and requires additional analysis for the interface between languages to support multi-language programs.

Finally, union slicing (Beszédes et al., 2002) is also related to ORBS. Union slicing approximates a static slice by unioning dynamic slices obtained using a set of inputs. However, union slicing inherits the critical difference between dynamic and observationbased slicing: dependencies considered by union slicing are dynamically occurring (but statically determined) dependencies, rather than dynamically observed dependencies as in ORBS. Moreover, unioning of slices does not necessarily lead to correct slices (De Lucia et al., 2003), whereby ORBS computes dynamic slices for multiple criteria 
without unioning.

MOBS builds upon Observation-Based Slicing (ORBS), a type of dynamic slicing: it only preserves program dependencies that are observable (Binkley et al., 2014a) via program execution. The dynamic nature of ORBS means it under-approximates the semantics of program dependence, limited by the test suites used as input. However, accepting deletions of source code lines based on purely dynamic observation has its own benefits, such as being able to handle dependencies that no static slicers can cope with (Binkley et al., 2015), slicing multi-lingual systems (Binkley et al., 2015), and slicing languages with highly unconventional program semantics such as Picture Description Language (Yoo et al., 2017). While MOBS and ORBS uses deletions of source code lines, a later variant (Gold et al., 2017; Binkley et al., 2019) represents source code as a tree structure and the proceeds to delete subtrees. Binkley et al. (2014a) also introduced a parallel ORBS. Rather than applying window deletion successively, the parallel version applies all deletion operators of different window size in parallel and then selects the largest deletion that succeeds. By definition, MOBS is very much parallelisable. Furthermore, if the deletion operators MOBS uses subsume all the window deletion operators used by W-ORBS, parallel MOBS is a super set of parallel $\mathrm{W}-\mathrm{ORBS}$. Theoretically, its worst case performance will match that of parallel WORBS, but it has the potential to opportunistically take advantage of successful lexical deletions. In this paper, we focus evaluating the impact of lexical dependence; thus, we leave the study of parallelisation's impact to future work.

The notion of deleting parts of a program or inputs also features prominently in Delta Debugging (Zeller, 1999; Cleve and Zeller, 2000; Zeller and Hildebrandt, 2002). Some variants of delta debugging try to reduce the cost of the original Delta Debugging by exploiting language syntax and semantics. For example, Hierarchical Delta Debugging (Misherghi and Su, 2006) exploits tree structures providing a tree-based Delta Debugging approach. Delta (McPeak et al., 2006), a well known implementation of Delta Debugging, uses a separate tool to flatten the tree structures in source code, before applying delta debugging. Regehr et al. (2012) exploit the syntax and semantics of $\mathrm{C}$ for four delta-debugging based algorithms to minimize $\mathrm{C}$ programs that trigger compiler bugs. Coarse Hierarchical Delta Debugging (Hodován et al., 2017) is a recently introduced variant of Hierarchical Delta Debugging that filters out tree nodes that are not allowed to be deleted by the grammar of the language, thereby speeding up Hierarchical Delta Debugging.

Finally, Jiang et al. (2014) introduced a forward dynamic slicing approach similar to ORBS: their technique mutates the value of the variable at the location of the slicing criterion, and subsequently observes the computed values in the state trajectory. The dynamic slice consists of all statements for which the computed values have changed compared to the trajectory of the original program.

\section{Conclusion}

Given program slicing's wide range of applications, an efficient, language-independent slicing technique can bring significant benefits to developers. The small increase in slice size produced by MOBS is likely acceptable if it is accompanied by a significant decrease in slicing time. 
This paper makes two novel technical contributions. First, we present a novel generalisation of observational slicing that can take advantage of a wide range of deletion operators rather than the original algorithm's use of only one, the deletion window. Second, we introduce lexical deletion operators that exploit lexical similarities between source code lines to improve the efficiency of ORBS. MOBS is the resulting observational slicer that uses multiple deletion operators including the existing deletion window operators and the newly-introduced lexical deletion operators.

The results of our empirical evaluation of MOBS show a significantly improve in efficiency over W-ORBS, which is based solely on window deletion: MOBS deletes approximately $69 \%$ of the lines deleted by W-ORBS, while taking only about $36 \%$ the wall clock execution time. Furthermore, ROS's ability to learn the relative applicability of different operators dynamically during slicing produced the best result for MOBS. Finally, we qualitatively considered the lexically deletable lines of code and the scalability of MOBS using the multi-lingual open-source project misaka, which include both Python and $\mathrm{C}$ code.

These results show that using a lexical approximation of dependence is viable. Future work will include investigating the impact of involving reserved words and comments in the lexical similarity computations as well as the impact of increasing the size of the test corpus and programs.

\section{Acknowledgement}

This research was supported by Next-Generation Information Computing Development Program through the National Research Foundation of Korea (NRF) funded by the Ministry of Science, ICT (No. 2017M3C4A7068179). Dr. Binkley is supported by NSF grant 1626262.

\section{References}

Agrawal, H., DeMillo, R.A., Spafford, E.H., 1993. Debugging with dynamic slicing and backtracking. Software Practice and Experience 23, 589-616.

Agrawal, H., Horgan, J.R., 1990. Dynamic program slicing, in: Proc. of the ACM SIGPLAN'90 Conf. on Programming Language Design and Implementation (PLDI), pp. 246-256.

Allamanis, M., Barr, E.T., Bird, C., Sutton, C., 2014. Learning natural coding conventions, in: Proceedings of the 22Nd ACM SIGSOFT International Symposium on Foundations of Software Engineering, ACM, New York, NY, USA. pp. 281-293.

Amtoft, T., Banerjee, A., 2016. A Theory of Slicing for Probabilistic Control Flow Graphs. Springer Berlin Heidelberg, Berlin, Heidelberg. pp. 180-196.

Anderson, P., Teitelbaum, T., 2001. Software inspection using CodeSurfer, in: Workshop on Inspection in Software Engineering (CAV 2001).

Barpanda, S.S., Mohapatra, D.P., 2011. Dynamic slicing of distributed object-oriented programs. IET software 5, 425-433. 
Beszédes, Á., Faragó, C., Szabó, Z.M., Csirik, J., Gyimóthy, T., 2002. Union slices for program maintenance, in: Proc. of the 18th Intl. Conf. on Software Maintenance (ICSM), pp. 12-21.

Beszedes, A., Gergely, T., Gyimóthy, T., 2006. Graph-less dynamic dependencebased dynamic slicing algorithms, in: Intl. Workshop on Source Code Analysis and Manipulation (SCAM), pp. 21-30.

Beszedes, A., Gergely, T., Szabó, Z.M., Csirik, J., Gyimothy, T., 2001. Dynamic slicing method for maintenance of large $\mathrm{C}$ programs, in: Proc. of the 5th Conf. on Software Maintenance and Reengineering, pp. 105-113.

Binkley, D., Gold, N., Harman, M., Islam, S., Krinke, J., Yoo, S., 2014a. ORBS: Language-independent program slicing, in: Proceedings of the 22nd ACM SIGSOFT International Symposium on the Foundations of Software Engineering, pp. 109-120.

Binkley, D., Gold, N., Harman, M., Islam, S., Krinke, J., Yoo, S., 2015. ORBS and the limits of static slicing, in: Proceedings of the 15th IEEE International Working Conference on Source Code Analysis and Manipulation, pp. 1-10.

Binkley, D., Gold, N., Islam, S., Krinke, J., Yoo, S., 2019. A comparison of tree- and line-oriented observational slicing. Empirical Software Engineering 24, $3077 \hat{a} \mathrm{~A} S ̧ 3113$.

Binkley, D., Heinz, D., Lawrie, D., Overfelt, J., 2014b. Understanding LDA in source code analysis, in: Proceedings of the $22 \mathrm{Nd}$ International Conference on Program Comprehension, ACM. pp. 26-36.

Binkley, D.W., 1998. The application of program slicing to regression testing. Information and Software Technology Special Issue on Program Slicing 40, 583-594.

Blei, D.M., Ng, A.Y., Jordan, M.I., 2003. Latent dirichlet allocation. Journal of machine Learning research 3, 993-1022.

Campbell, B.A., Treude, C., 2017. NLP2Code: Code snippet content assist via natural language tasks. CoRR abs/1701.05648. URL: http://arxiv.org/abs/1701. Q5648.

Cleve, H., Zeller, A., 2000. Finding failure causes through automated testing, in: Intl. Workshop on Automated Debugging, pp. 254-259.

DeMillo, R.A., Pan, H., Spafford, E.H., 1996. Critical slicing for software fault localization, in: Proc. of the Intl. Symposium on Software Testing and Analysis (ISSTA), pp. 121-134.

Do, H., Elbaum, S.G., Rothermel, G., 2005. Supporting controlled experimentation with testing techniques: An infrastructure and its potential impact. Empirical Software Engineering 10, 405-435.

Gallagher, K.B., Binkley, D., Harman, M., 2006. Stop-list slicing, in: Intl. Workshop on Source Code Analysis and Manipulation (SCAM), pp. 11-20. 
Gallagher, K.B., Lyle, J.R., 1991. Using program slicing in software maintenance. IEEE Transactions on Software Engineering 17, 751-761.

Gold, N., Binkley, D., Harman, M., Islam, S., Krinke, J., Yoo, S., 2017. Generalized observational slicing for tree-represented modelling languages, in: Proceedings of the 11th Joint Meeting of the European Software Engineering Conference and the ACM SIGSOFT Symposium on the Foundations of Software Engineering, pp. 547-558.

Goldberg, D.E., 1989. Genetic Algorithms in Search, Optimization \& Machine Learning. Addison-Wesley, Reading, MA.

Harman, M., Danicic, S., 1997. Amorphous program slicing, in: $5^{\text {th }}$ IEEE International Workshop on Program Comprenhesion (IWPC'97), IEEE Computer Society Press, Los Alamitos, California, USA. pp. 70-79.

Hindle, A., Barr, E.T., Su, Z., Gabel, M., Devanbu, P., 2012. On the naturalness of software, in: Proceedings of the 34th International Conference on Software Engineering, IEEE Press, Piscataway, NJ, USA. pp. 837-847.

Hodován, R., Kiss, Á., Gyimóthy, T., 2017. Coarse hierarchical delta debugging, in: 2017 IEEE International Conference on Software Maintenance and Evolution (ICSME), pp. 194-203. doi:10.1109/ICSME.2017.26.

Horwitz, S., Reps, T., Binkley, D.W., 1990. Interprocedural slicing using dependence graphs. ACM Transactions on Programming Languages and Systems 12, $26-61$.

Hur, C.K., Nori, A.V., Rajamani, S.K., Samuel, S., 2014. Slicing probabilistic programs, in: Proceedings of the 35th ACM SIGPLAN Conference on Programming Language Design and Implementation, ACM, New York, NY, USA. pp. 133-144.

Jiang, S., Santelices, R., Grechanik, M., Cai, H., 2014. On the accuracy of forward dynamic slicing and its effects on software maintenance, in: Intl. Working Conf. on Source Code Analysis and Manipulation (SCAM), pp. 145-154.

Korel, B., Laski, J., 1988. Dynamic program slicing. Information Processing Letters $29,155-163$.

Korel, B., Laski, J., 1990. Dynamic slicing in computer programs. Journal of Systems and Software 13, 187-195.

Korel, B., Rilling, J., 1998. Program slicing in understanding of large programs, in: $6^{\text {th }}$ IEEE International Workshop on Program Comprenhesion (IWPC'98), IEEE Computer Society Press, Los Alamitos, California, USA. pp. 145-152.

Krinke, J., 2003. Barrier slicing and chopping, in: IEEE International Workshop on Source Code Analysis and Manipulation (SCAM 2003), IEEE Computer Society Press, Los Alamitos, California, USA. pp. 81-87.

Le, T.B., Thung, F., Lo, D., 2014. Predicting effectiveness of IR-based bug localization techniques, in: 25th IEEE International Symposium on Software Reliability Engineering, ISSRE 2014, Naples, Italy, November 3-6, 2014, pp. 335-345. 
Le, T.D.B., Oentaryo, R.J., Lo, D., 2015. Information retrieval and spectrum based bug localization: Better together, in: Proceedings of the 2015 10th Joint Meeting on Foundations of Software Engineering, ACM, New York, NY, USA. pp. 579-590.

De Lucia, A., Harman, M., Hierons, R., Krinke, J., 2003. Unions of slices are not slices, in: European Conference on Software Maintenance and Reengineering (CSMR 2003), pp. 363-367.

McPeak, S., Wilkerson, D.S., Goldsmith, S., 2006. Delta (http://delta.tigris . org). URL: http://delta.tigris.org.

Misherghi, G., Su, Z., 2006. HDD: hierarchical delta debugging, in: Proc. of the 28th Intl. Conf. on Software Engineering (ICSE), pp. 142-151.

Mitra, M., Chaudhuri, B., 2000. Information retrieval from documents: A survey. Information Retrieval 2, 141-163.

Mund, G., Mall, R., 2006. An efficient interprocedural dynamic slicing method. Journal of Systems and Software 79, 791-806.

Orso, A., Sinha, S., Harrold, M.J., 2001. Incremental slicing based on data-dependences types, in: Proceedings of the IEEE International Conference on Software Maintenance (ICSM 2001), IEEE Computer Society Press, Los Alamitos, California, USA. pp. 158-167.

Panichella, A., Dit, B., Oliveto, R., Di Penta, M., Poshyvanyk, D., De Lucia, A., 2013. How to effectively use topic models for software engineering tasks? an approach based on genetic algorithms, in: Proceedings of the 2013 International Conference on Software Engineering, IEEE Press, Piscataway, NJ, USA. pp. 522-531.

Ragkhitwetsagul, C., Krinke, J., Clark, D., 2018. A comparison of code similarity analysers. Empirical Software Engineering 23, 2464-2519.

Rajaraman, A., Ullman, J.D., 2011. Mining of Massive Datasets. Cambridge University Press, New York, NY, USA.

Regehr, J., Chen, Y., Cuoq, P., Eide, E., Ellison, C., Yang, X., 2012. Test-case reduction for $\mathrm{C}$ compiler bugs, in: Proc. of the ACM SIGPLAN Conf. on Programming Language Design and Implementation (PLDI), pp. 335-346.

Saha, R.K., Lease, M., Khurshid, S., Perry, D.E., 2013. Improving bug localization using structured information retrieval, in: Automated Software Engineering (ASE), 2013 IEEE/ACM 28th International Conference on, IEEE. pp. 345-355.

Salton, G., Wong, A., Yang, C.S., 1975. A vector space model for automatic indexing. Commun. ACM 18, 613-620.

Singhal, A., 2001. Modern information retrieval: A brief overview. IEEE Data Engineering Bulletin 24, 35-43. 
Szegedi, A., Gyimóthy, T., 2005. Dynamic slicing of Java bytecode programs, in: Intl. Workshop on Source Code Analysis and Manipulation (SCAM), pp. 35-44.

Vargha, A., Delaney, H.D., 2000. A critique and improvement of the "CL" common language effect size statistics of McGraw and Wong. Journal of Educational and Behavioral Statistics 25, pp. 101-132.

Wang, Q., Parnin, C., Orso, A., 2015. Evaluating the usefulness of IR-based fault localization techniques, in: Proceedings of the 2015 International Symposium on Software Testing and Analysis, ISSTA 2015, Baltimore, MD, USA, July 12-17, 2015, pp. 1-11.

Weiser, M., 1979. Program slices: Formal, psychological, and practical investigations of an automatic program abstraction method. Ph.D. thesis. University of Michigan, Ann Arbor, MI.

Weiser, M., 1981. Program slicing, in: Proc. of the 5th Intl. Conf. on Software Engineering, pp. 439-449.

Yoo, S., Binkley, D., Eastman, R., 2017. Observational slicing based on visual semantics. Journal of Systems and Software 129, 60-78.

Zeller, A., 1999. Yesterday, my program worked. today, it does not. Why?, in: European Software Engineering Conf. and Foundations of Software Engineering, pp. 253-267.

Zeller, A., Hildebrandt, R., 2002. Simplifying and isolating failure-inducing input. IEEE Transactions on Software Engineering 28, 183-200.

Zhang, X., Gupta, N., Gupta, R., 2007. A study of effectiveness of dynamic slicing in locating real faults. Empirical Software Engineering 12.

Zhang, X., Gupta, R., 2004. Cost effective dynamic program slicing, in: Proc. of the ACM SIGPLAN 2004 Conf. on Programming Language Design and Implementation, pp. 94-106. 